\title{
La tavolozza del poeta: l'arte della comunicazione iconica in un dialogo di Ludovico Dolce
}

\author{
Susanna Villari \\ Università degli Studi di Messina \\ susanna.villari@unime.it
}

\section{Riassunto}

L'articolo propone un'analisi del Dialogo dei colori di Ludovico Dolce, evidenziando non solo gli agganci con l'emblematica e con l'arte della comunicazione iconica, ma anche i fili di un dibattito culturale che prelude alla poetica del Barocco.

Parole chiave: Ludovico Dolce; Dialogo dei colori; Cinquecento; comunicazione iconica; emblemi.

Abstract. The poet's palette: the art of iconic communication in a dialogue by Ludovico Dolce

The article proposes an analysis of Ludovico Dolce's Dialogo dei colori and highlights the links with the art of emblems and of iconic communication, as well as the thread of the cultural debate that preluded the Baroque's poetics.

Keywords: Ludovico Dolce; Dialogo dei colori; Sixteenth century; iconic communication; emblems. 
Nella vastissima produzione di Ludovico Dolce si annovera un Dialogo dei colori $^{1}$ che attende ancora attenzioni critiche e filologiche. La scarsa considerazione di questo opuscolo è con tutta probabilità ascrivibile al suo carattere divulgativo e di rivisitazione, ai limiti tra il volgarizzamento, il rifacimento e il plagio, di precedenti trattati omologhi di maggiore successo: ${ }^{2}$ in particolare il De coloribus del cosentino Antonio Telesio $(1528)^{3}$ e il Del significato de' colori dell'umanista ferrarese Fulvio Pellegrino Morato (1535), quest'ultimo corredato nelle edizioni successive (a partire dal 1543) di un ampio repertorio finale sul significato di fiori, erbe e altri elementi. ${ }^{4}$

Il Dialogo dei colori non manca comunque di essere citato e osservato con una certa curiosità nell'ambito degli studi moderni sulla trattatistica dedicata al colore, nonché nell'ambito dell'itinerario culturale di Dolce; ${ }^{5}$ egli fu anche autore di quel Dialogo della pittura, edito nel 1557, il cui ruolo di rilievo nel panorama culturale del tempo è oggi ampiamente riconosciuto, soprattutto per l'assunzione del motivo cromatico quale cardine per valorizzare la pittura di Tiziano e l'arte veneziana in alternativa al paradigma michelangiolesco promosso da Vasari. ${ }^{6}$ Vale la pena ricordare che nell'ambiente artistico-culturale lagunare del suo secolo l'intellettuale veneziano aveva svolto il ruolo di promotore culturale, di interprete e mediatore delle tendenze letterarie e artistiche, insistendo fin dalla sua produzione giovanile sull'interazione tra poesia e arti figurative, con il topos del poeta imitatore con penna e parole e del pittore imitatore con pennelli e colori. Il Dialogo della pittura attuava l'interpretazione in

1. Dolce, L. (1565a): vd. frontespizio e colophon, infra, fig. 9 e 14. Si legge anche nell'edizione curata da D. Ciàmpoli (Dolce, L., 1913), priva di commento e della Tavola finale dell'opera, su cui infra, fig. 11-13. Una parziale trascrizione dell'opera, preceduta da una breve premessa di Luisa Gasparotto, anche on line: Dolce, L. (2014).

2. Per analoghe ragioni parte della produzione di Dolce risulta del resto ancora poco valorizzata, sebbene nuove prospettive di studio e ricerca si siano aperte negli ultimi decenni, anche sul versante critico-editoriale. Si segnala, tra l'altro, il volume miscellaneo dedicato a Dolce letterato (Marini, P. \& Procaccioli, P., edd., 2016).

3. Telesio, A. (1528). Si legge, con relativo commento, in Osborne, R. (2019), pp. 297-308 e 99-110, per la traduzione in lingua inglese. L'edizione parigina del trattato (presso Roberto Stefano, 1549) è oggi disponibile in ristampa anastatica: Telesio, A. (2010), su cui cfr. la recensione di Collin, F. (2011).

4. L'editio princeps dell'opera reca il titolo Del significato de' colori (Morato, F.P., 1535), su cui si fonda l'edizione, anche in versione inglese, a cura di Osborne, R. (2019), pp. 153-158, 309-333; la seconda edizione, intitolata Del significato de colori e de mazzolli, è ampliata con un corollario finale (Morato, F.P., 1543); utilizzo quest'ultima per le citazioni. Sull'autore: Saracco, L. (2012).

5. Cfr. Cian, V. (1894), Salza, A. (1901), Pozzi, M. (1968, pp. 258-259, nota 1), Terpening, R. (1997, pp. 158-164 e note), Torre, A. (2001) e Osborne, R. (2019; per Dolce, ad indicem).

6. Dolce, L. (1557). Vasta la bibliografia sul Dialogo della pittura, per la quale rinvio a Arroyo Esteban, S. (2016) e alla sua edizione del trattato (Dolce, L, 2010), nonché alle altre edizioni moderne corredate di introduzioni e commenti (Dolce, L., 1968 e Dolce, L., 1996).

7. Cfr. almeno Dolce, Apologia contra ai detrattori dell'Ariosto, in Orlando furioso (Venezia, Pasini e Bindoni, 1535); cfr. Villari, S. (2013), pp. 152-165, in part. p. 161; Dolce, Osservationi nella volgar lingua (Dolce L., 1550, cc. $87 v$-88v; Dolce, L., 2004, pp. 470-472); Dolce, lettera a Gasparo Ballini (1554/55) (ora in Dolce, L., 2015, pp. 142-148, in part. 
chiave aristotelica delle opere d'arte figurativa, applicando ai dipinti gli stessi parametri di giudizio utilizzati per i testi letterari e rendendo fertile così un terreno di indagine già dissodato da Francesco Robortello e Vincenzo Maggi, nel contesto dei commenti alla Poetica aristotelica, e anche da Benedetto Varchi nelle sue Due lezzioni. ${ }^{8}$

Il Dialogo della pittura e il Dialogo dei colori, nonostante l'apparente complementarità tematica e la scelta della forma dialogica, sono tuttavia ben distanti per ambiti, consistenza e intenti. Il libretto dei colori, infatti, va riportato al contesto specifico dell'ecfrasi, dell'emblematica, dei geroglifici, dell'arte delle imprese e dei percorsi della memoria, ${ }^{9}$ in conformità con altri trattati sugli stessi temi. Il retroterra culturale va dunque individuato in quel genere di letteratura che affonda le sue radici nei Hieroglyphica di Horapollo (tradotti, o meglio rielaborati, da Pierio Valeriano nel 1556), ${ }^{10}$ approdando ad opere come l'Hypnerotomachia Poliphili di Francesco Colonna (1499), gli Emblemata di Andrea Alciato (1531), il Dialogo delle imprese militari e amorose di Paolo Giovio (edito nel 1555) e i Symbolicarum quaestionum libri quinque di Achille Bocchi (pure 1555). ${ }^{11}$

A dispetto del titolo, all'interno del Dialogo dei colori di Dolce si individuano almeno due ambiti distinti della trattazione: i colori e i loro significati; gli oggetti e i loro significati. Eppure tra l'uno e l'altro non vi è soluzione di continuità: con una tecnica a incastro, Dolce riesce a legare, traducendo o mutuando in vari casi alla lettera, il dettato dei suoi predecessori. E infatti ricalca il libretto di Telesio (sulla classificazione dei colori) e ne assume l'epilogo come avvio della trattazione successiva, modellata sull'opuscolo di Morato (sul significato dei colori); e ancora sfrutta la conclusione di quest' ultimo come premessa alle argomentazioni, che appaiono in varia misura originali, sulla simbologia degli oggetti e dei doni. ${ }^{12}$

p. 146). Per l'attenzione di Dolce agli aspetti ecfrastici delle opere letterarie e alla loro resa iconografica cfr. Genovese, G. \& Torre, A. (2019), ad indicem.

8. Robortello, F. (1548); Maggi, V. - Lombardi, B. (1550); Varchi, B. (1549). Cfr. Sgarbi, M. (2015), anche per la bibliografia, cui aggiungerei, per il rilievo dato a Ludovico Dolce, Padoan, G. (1978) e, per le lezioni di Varchi, in questo stesso fascicolo, Tramontana, A. (2021).

9. A Dolce si deve anche un Dialogo nel quale si ragiona del modo di accrescere e conservar la memoria (Dolce, L., 1562), nel quale traspone il contenuto del Congestorium artificiosae memoriae di Johannes Host von Romberch (Venezia, Melchiorre Sessa, 1553). Fulcro dell'opera è l'idea che la tecnica di creazione delle immagini, propria dei poeti e dei pittori, è funzionale alla memoria e alla conoscenza e che le stesse immagini sono portatrici di sapere (cfr. Bolzoni, L., 1995, per Dolce ad indicem). Il trattato di Dolce si legge oggi nell'edizione a cura di Andrea Torre (Dolce, L., 2001), a cui si rinvia per la ricca introduzione, dove Dialogo della pittura, Dialogo della memoria, Dialogo dei colori vengono osservati nelle loro correlazioni come parti di un preciso progetto culturale (in particolare: Torre, A., 2001, pp. XXXVII-XLI).

10. Cfr. Valeriano, P. (1556).

11. Per una efficace sintesi delle sollecitazioni culturali scaturite dalla scoperta del manoscritto di Horapollo e sulla fortuna di questa enigmatica opera: Doglio, M.L. (1978) e Gabriele, M. (2021), in part. pp. 40-41.

12. Coronato Occolti, qualche anno più tardi, introduce una sezione specifica sul «Significato 
Risulta di qualche utilità indagare la trattatistica del colore anche in una dimensione collettiva, evidenziando l'intertestualità senza offuscare gli apporti individuali degli autori, mettendo a fuoco le comuni matrici classiche, gli agganci alla tradizione, la convergenza di obiettivi ed esiti, e in sostanza le ragioni più profonde dell'interesse per il tema cromatico. ${ }^{13}$

Dolce, in particolare, ripropone l'altrui dettato, traduce, semplifica, filtra e integra i testi attraverso le proprie competenze e conoscenze, obbedendo del resto a una strategia divulgativa consona alla propria fisionomia intellettuale. Strategia da valutarsi entro il suo proprio sistema, alla luce dei meccanismi della diffusione culturale ed editoriale del Cinquecento, e senza il condizionamento dei nostri attuali valori e criteri di giudizio sulla proprietà letteraria $\mathrm{e}$ sul dovere di originalità nella produzione poetica e artistica, a maggior ragione in una trattatistica così specializzata. ${ }^{14}$

Indicazioni basilari sul Dialogo dei colori di Dolce si traggono dai paratesti e dalle soglie del testo: in particolare la dedica al giureconsulto veronese Agostino Brenzone (nella quale è istituito il rapporto tra materialità dei colori e colori "della eloquenza", Dolce, 1565a, cc. A2r-3v); l'avvertenza editoriale "Ai lettori" (ivi, cc. $4 r-5 v$ ), dove si dichiara il distacco dalla materia specifica del colore in favore di disquisizioni su argomenti di vario genere e varia utilità; l'esordio dell'opera, dove si delinea l'occasione del dialogo (ivi, c. $6 r$ e sgg.); l'indice finale, intitolato "Tavola dei colori", che accoglie di fatto non soltanto nomi di colori, ma in maggioranza di cose (fiori, frutti, piante, erbe, animali, pietre preziose, utensili) e di personaggi mitici e storici (ivi, cc. $87 r-88 r$ e fig. $11-13) .{ }^{15}$

di alcuni doni» nel suo Trattato dé colori (Occolti, C., 1568, cc. 61r-77v).

13. Tali criteri di metodo sono deducibili in ultima analisi dagli studi di Roy Osborne (2019), il quale, con un ampio sguardo anche all'orizzonte europeo, accoglie in un serrato confronto i testi (integrali o parziali, in originale e in traduzione) delle opere di Jean Courtois (Le Blason de toutes armes et éscutz, 1495), Gilles Corrozet (Le Blason des couleurs en armes, livrées et devises, 1527), Antonio Telesio (Libellus de coloribus, 1528), Fulvio Pellegrino Morato (Del significato de' colori, 1535), Gerard Leigh (The Accedens of Armory, 1562), Giovanni Paolo Lomazzo (Trattato dell'arte della pittura, scoltura et architettura, 1584), rintracciando le fonti letterarie, nonché il rapporto tra simbolismo dei colori e simbolismo araldico. Tuttavia un campione significativo, e anche più ampio, di testi era stato offerto da Paola Barocchi (1971-1977), vol. II, pp. 2125-2324 (selezione dai trattati di Leonardo, Mario Equicola, Fulvio Pellegrino Morato, Giorgio Vasari, Coronato Occolti, Lodovico Dolce, Giovan Paolo Lomazzo, Giovan Battista Armenini, Giovanni de' Rinaldi, Antonio Calli). Il commento della Barocchi disegna nell'insieme una mappa di rapporti intertestuali.

14. Per queste operazioni di traductio, nel senso ampio di "trasferimento di strutture, di concetti e di forme, che spazia dal grado minimo della trasposizione parola per parola a quello massimo della riscrittura”: Torre, A. (2001), p. X. Helena Sanson, inoltre, definisce l'operato di Dolce con l'ossimoro "Original plagiarism" (Sanson, H., 2015, p. 1).

15. L'opera è presentata quale "selva di varie lettioni" nella premessa editoriale "Ai lettori" (Dolce, 1565a, c. $4 v$ ), dove, tra l'altro, sono preannunciate altre due opere dello stesso autore (un "Trattatello intorno alla proprietà delle gemme" e un "Sommario di tutta la Filosofia di Aristotele"), uscite nello stesso anno e destinate, insieme al trattato dei colori, a formare un trittico di grande utilità: cfr. Dolce, L., (1565b) e [1565c]. 
Appropriandosi, per la prima sezione del suo dialogo, del testo di Telesio, Dolce parte comunque dal colore, osservato come uno dei tanti aspetti di una realtà variegata e multiforme, suscettibile di molteplici interpretazioni. Ponendosi, in linea con il letterato cosentino, nell'ottica di "uno, il cui studio è di lettere e non di pittura" (ivi, c. 7r), egli analizza i termini capaci di esprimere e rappresentare le innumerevoli sfumature dei colori, prodotti dalla natura o artificiosamente creati, offrendo dunque a chi legge e a chi scrive gli strumenti compositivi o di valutazione degli aspetti ecfrastici delle opere letterarie.

L'aderenza al libretto di Telesio ha precise conseguenze, in quanto la virtuale 'tavolozza del poeta' si arricchisce di una nomenclatura estranea alla tradizione poetica nostrana: accanto ai colori canonici, di norma presenti in segmenti ecfrastici dei 'classici' in volgare, Dolce annovera una serie terminologica presa in prestito dal lessico latino o a volte greco (veri e propri calchi, come antracino, aquilo, atro, cesio, cianeo, cilestre, ferrugineo, furvo, glauco, prasino, pullo, puniceo, e altri passati sistematicamente in rassegna per una disamina anche etimologica o pseudo-etimologica); a colpo d'occhio si tratta di una complessa mappa lessicale cromatica, soltanto occasionalmente sostenuta da esempi concreti di uso letterario. È possibile però intravedere in filigrana il lessico cromatico dell' Hypnerotomachia Poliphili di Francesco Colonna, opera che, dopo la prestigiosa edizione aldina del 1499, circolò anche nella ristampa veneziana, a cura degli eredi di Aldo, del 1545. Essa non è citata dai trattatisti sul colore, ma è molto probabile che abbia esercitato forti suggestioni per le funzioni evocative di un lessico ingegnoso, costruito secondo i parametri delle raffigurazioni artistiche, ovvero equiparando il rapporto tra le parole all'accostamento di segni e colori, ${ }^{16}$ con l'esito di un peculiare impasto linguistico. Questa ipotesi di lavoro può essere sostenuta già da qualche veloce affondo, verificando l'uso da parte del Colonna di aggettivi come anhtracino (Colonna, F., 1980, pp. 274, 327), caesio (ivi, p. 328), furvo (ivi, p. 230), ferrugineo (ivi, p. 326), praxino (ivi, pp. 42, 310,326), pullo (ivi, p. 400), e tanti altri, le cui variegate implicazioni semantiche possono di contro trovare a loro volta ragioni e conferme proprio nella trattatistica sul colore, che filtra e rielabora le informazioni dell'antichità ricostruendone alcuni percorsi eruditi.

Una terminologia di questo tipo, coerente nella sua specificità all'interno del trattato latino di Telesio, ma anche conforme alle qualità della prosa mescidata ed impressionistica di Colonna, colpisce all'interno del Dialogo di Dolce, proprio in quanto irrelata rispetto alla tradizione letteraria e poetica in volgare alla quale l'autore intende riferirsi.

Dolce dichiara comunque in esordio i propri debiti con il De coloribus di Telesio, non senza far implicito riferimento ad altri testi antichi e moderni:

16. Secondo Giovanni Pozzi (Premessa, in Colonna, F., 1980, vol. II, p. 13*), la lingua di Colonna possiede "il quid della pittura intesa come composizione di vari colori, delle luci e delle ombre [...]". Mi avvalgo di questa edizione, anche per l'utile glossario (vol. II, pp. 269-308), ma tengo conto anche dell'introduzione e del commento dell'edizione successiva, corredata di ristampa anastatica, a cura di Marco Ariani e Mino Gabriele (Colonna, F., 1998). 
[...] essendomi venuto questi dì alle mani un libricciuolo tra molti, che ne ho altre volte di diversi letto, così antichi come moderni, di M. Antonio Tilesio da lui latinamente scritto; il qual Tilesio fu huomo di belle lettere e di fin giudicio, e scrisse in questa materia assai acconciamente, valendomi di quanto così alla sfuggita posso ricordarmi: che sia, però, s'io non m'inganno a bastanza. ${ }^{17}$

La lettura del trattatello latino si aggiungeva, pertanto, a supporto delle conoscenze deducibili da altri trattati, sollecitando una rinnovata divulgazione della materia. Si può del resto presupporre, da parte dei trattatisti cinquecenteschi, l'ideale riconoscimento del De coloribus quale archetipo del genere (il primo a introdurre una visuale prettamente letteraria). Proprio dalla premessa dello scrittore cosentino si traggono i criteri di base ai quali i letterati potevano ispirarsi:

Dicam aliquid de coloribus in hoc libello, non quidem unde conficiantur aut quae sit eorum natura, neque enim Pictoribus haec traduntur, aut Philosophis, sed tantum Philologis, qui latini sermonis elegantiam studiose inquirunt. Scribam omnia breviter et accurate ac rerum ipsarum nomina, quo statim colores intelligantur, singulis apponam (Telesio, I 528 , c. a3r).

A partire dal volgarizzamento del trattato di Telesio, la questione del colore veniva recuperata come fulcro di una riflessione su una realtà da rappresentare e da interpretare con gli strumenti della letteratura. Ma ciò - come si deduce dal dialogo di Dolce - doveva comportare l'esigenza da un lato di allargare il campo d'indagine, non rinunciando all'osservazione di quanto programmaticamente estraneo al libello telesiano (l'esame dell'essenza e della natura del colore) e dall'altro di rimodulare i rinvii alla tradizione latina con esempi propri della letteratura in volgare italiano.

La scelta della struttura dialogica - elemento distintivo rispetto ad altri trattati omologhi (tra i quali quelli di Telesio e Morato) - risulta perfettamente funzionale allo sviluppo argomentativo, pur lasciando trasparire, come è stato notato, l'originale impianto trattatistico dell'antigrafo. ${ }^{18}$ I due interlocutori (non identificati) assolvono due ruoli diversi: l'uno, Mario, pone interrogativi, impersonando il lettore di media cultura, curioso e interessato a colmare le lacune delle proprie conoscenze; l'altro, Cornelio, alter ego dell'autore, onnivoro produttore e divulgatore culturale, risponde in modo essenziale e sintetico, evitando eccessi dottrinari. L'esito è quello di una naturale conversazione dotta tra amici, con divagazioni dal tema principale e con escursioni di registro, pur mantenendo costanti gli obiettivi morali e pedagogici. ${ }^{19}$

17. Dolce, L. (1565a), c. $6 v$. Per le citazioni di questo e di altri testi coevi mi limito a una normalizzazione paragrafematica.

18. La trasposizione in forma dialogica del contenuto del modello riguarda allo stesso modo il Dialogo della memoria e il Dialogo della institution delle donne (Dolce, L., 1545; id., 2015), quest'ultimo fondato sul De institutione foeminae christianae di Juan Luis Vives (Antwerp: Hoochstratanum, 1524). Cfr. Torre, A. (2001), pp. XXVIII-XXXV; Sanson, H. (2015), pp. 39-40.

19. Cfr. l'avviso "Ai Lettori" (Dolce, L., 1565a), c. 4 rv. 
Mario esordisce con precisi interrogativi, che impongono risposte e definizioni altrettanto precise: "cosa sia colore, quante sorti di colori si trovino, e la proprietà e il significato loro" (Dolce, 1565a, c. 6v). Dopo il preambolo pseudoscientifico sulla qualità del colore, ${ }^{20}$ Dolce, sulla falsariga di Telesio, si sofferma sulla nomenclatura coloristica, con una ricerca etimologica mirata a ricostruire la connotazione degli aggettivi, a classificarli, a distinguerli all'interno degli stessi campi semantici, per individuare il lessico cromatico idoneo a descrivere l'impatto visivo degli stessi oggetti in situazioni dissimili (il cielo, ad esempio, assume coloriture diverse a seconda delle condizioni climatiche). Nel testo tradotto letteralmente da Telesio, Dolce innesta brani tratti dalla tradizione in volgare, conferendo in tal modo rinnovata veste ai concetti tradotti dal trattato del cosentino. Basti ricordare qualche caso. Proprio a proposito del ceruleo, colore del cielo sereno ("proprie color est coeli, sed sereni", Telesio, A., 1528, c. a3r), per rendere l'aggettivo serenus Dolce ricorre a una perifrasi desunta da un emistichio della petrarchesca canzone "delle visioni" (cfr. Rvf, CCCXXIII, 17): "ceruleo [...] è propriamente il color del cielo, quando, come dice Petrarca, nulla nube il vela" (Dolce, 1565a, c. 9r). Inoltre, nell'esporre la varietà delle sfumature cromatiche e terminologiche, in aggiunta al dettato di Telesio (1528, c. a3 $v$ ), è richiamata la descrizione petrarchesca di un lembo del manto di Laura (Rvf, CLXXXV, 9-11: Dolce, 1565a, c. 9v). E ancora, per illustrare il colore cesio, Dolce ricorda non solo la descrizione virgiliana di Caronte (già presente in Telesio, 1528, c. b1 $r$ : "Stant circum lumina flamma"; cfr. Virgilio, Aen., VI, 300) ma anche le raffigurazioni di Dante (Inf., III, 109111: "Caron dimonio con occhi di bragia", ecc.) e di Michelangelo nel Giudizio della Cappella Sistina (Dolce, 1565a, cc. $10 v-11 r$ ). ${ }^{21}$ La disquisizione sul colore atro riceve come corollario l'interpretazione di una terzina petrarchesca (Tr. Cup., II, 142-44), relativa alla tonalità scura, e perciò non apprezzata, della pelle e dei capelli di Andromeda (Dolce, 1565a, c. 12r). Di seguito, di contro, la purezza del bianco trova espressione in due versi rispettivamente di Petrarca (Tr. Pud., 118) e di Bembo (Asolani, III, viii, 49). Il colore del ferro (o ferrugineo) è identificato con il "perso" del primo verso della canzone petrarchesca XXIX (Verdi panni sanguigni oscuri o persi). ${ }^{22}$

20. Dolce (1565a, cc. $7 r-8 v$ ) analizza le diverse concezioni del colore: quella pitagorica (colore come "superficie"), quella platonica (colore come "lume", Timeo, 67-68), quella aristotelica (colore come "lucidezza", Aristotele, De sensu et sensib., 437a sgg.), aderendo al concetto aristotelico di colore come "termino et estremità di lucido e terminato corpo" (Dolce, 1565 a, c. $7 r$ ). Sulla stessa linea si pone Raffaello Borghini nel trattato Il riposo (dedicato alla scultura e alla pittura: Borghini, R. (1584), c. 227. Cfr. Barocchi, P., (1971-1977), vol. II, p. 2212, nota 4 .

21. Sul Giudizio michelangiolesco, termine di confronto anche per l'elaborazione delle teorie artistiche di Dolce: Arroyo Esteban, S. (2015). Cfr. anche Torre, A. (2001), p. 36, nota 98 Per le implicazioni dell'associazione Dante / Michelangelo rinvio a Tramontana, A. (2021), in questo stesso fascicolo.

22. Sull'interpretazione cinquecentesca di questa canzone e in particolare sulla lettura di Benedetto Varchi: Girardi, M.T. (2005). Per Varchi, sulla scorta del Convivio dantesco, il "perso" è "misto di purpureo e nero" (ivi, p. 699). 
In altri casi Dolce traduce dal latino versi citati da Telesio. Degna di nota, ad esempio, è la sua trasposizione in trentadue endecasillabi sciolti dei diciotto versi coriambi di Telesio relativi alla leggenda dell'invenzione della porpora da parte di Ercole (Telesio, 1528, c. c[4]v; Dolce, 1565a, c.18rv). Lo scrittore cosentino li aveva a sua volta liberamente tradotti (ma senza dichiarazione esplicita) dall'Onomasticon del lessicografo greco Giulio Polluce (dunque con una versione inedita, dato che prima del 1541 l'opera si leggeva solo in lingua greca). ${ }^{23}$ Sulla scorta di Telesio, Dolce così diffondeva una leggenda peregrina, ma banalizzandone e semplificandone il dettato, poiché nella sua traduzione è attribuito a Ercole (e non al suo cane) il morso inferto al mollusco (murice o porpora) da cui sgorga il sangue destinato entrare nella storia del costume per le sue proprietà coloranti.

Talvolta Dolce sorvola sui versi latini riportati nel suo antecedente. Ad esempio, nella trasposizione del capitolo sul verde, egli tralascia i versi di un epigramma latino ("de scarabaeis iocosum epigramma"), che Telesio riferisce di aver composto ispirandosi alle caratteristiche dello scarabeo, dai cui preziosi colori e riflessi argentei veniva desunto, a suo dire, l'uso cosentino di denominare il coleottero "equus lunae" ("a nobis Cosentinis Equus Lunae nuncupatur", Telesio, 1528, c. c2v). Ebbene, non solo Dolce omette il testo dell'epigramma di Telesio, ma, nella sua trasposizione letterale ("da Consentini chiamato non senza convenevolezza cavallo di essa luna”, Dolce, 1565a, 16r) introduce il riferimento spurio e poco perspicuo ai cosentini, senza cogliere peraltro lo spunto per soffermarsi (neppure in altri luoghi del volumetto) sulla varia simbologia dello scarabeo, cui, ad esempio, Erasmo da Rotterdam aveva dedicato uno dei più noti e corposi dei suoi Adagia (Scarabaeus aquilam quaerit). ${ }^{24}$

Rispetto alla struttura in capitoli del modello latino, Dolce non inserisce cesure, ma scandisce il discorso mediante le battute di Mario oppure istituisce, per voce di Cornelio, relazioni mnemoniche o logiche tra un colore e un altro. ${ }^{25}$ L'epilogo di Telesio sulla varietà dei colori viene assunto infine dal poligrafo veneziano quale conclusione della prima sezione del suo dialogo (Dolce, 1565a, cc.16r-19v), suggellata da una battuta del personaggio Mario

23. L'editio princeps, con il solo testo greco, è del 1502 (Venezia, Aldo). Più tarda la versione latina: Iulii Pollucis Onomasticon, hoc est Dictionarium, nunc primum latinitate donatum, Rodolpho Gualthero Tigurino interprete, Basilea, Winter, 1541. Il brano in questione corrisponde a Onomasticon, I 45 (ed. Bekker, Berlin, Teubner, 1846).

24. Erasmo (1980), pp. 121-195. Per la storia della simbologia dello scarabeo in età medievale: Ciccarese, M.P. (2016).

25. Il De coloribus di Telesio è suddiviso in dodici capitoli (I: coeruleus; II: caesius; III: ater; IV: albus; V: pullus; VI: ferrugineus; VII: rufus; VIII: ruber; IX: roseus; X: puniceus; XI: fulvus; XII: viridis); a essi corrisponde nel trattato di Dolce la trattazione in sequenza dei medesimi colori (comprese le nomenclature secondarie): c. $9 r$ : ceruleo; c. $9 v$ : cesio; c. $11 r$ : atro; c. $12 r$ : bianco; c. $12 v$ : pullo; c. $13 r$ : ferrugineo; c. $14 r$ : rufo; c. $14 v$ : rubro; c. $15 r$ roseo; c. $15 r$ : puniceo; c. $15 v$ : fulvo; c. 16r: verde. Segue in entrambi l'epilogo (caput XIII in Telesio, seguito dai carmi Araneola e Cicindela), nel cui contesto si iscrive la leggenda sopra citata della scoperta della porpora. 
("Questo a me è a bastanza. Onde è tempo che tu entri a ragionar del significato de' colori”, Dolce, 1565a, c. 19v).

Ed è qui che entra in gioco il trattato di Pellegrino Morato, ripreso per ampie sezioni ${ }^{26}$ come Dolce implicitamente ammette attraverso le parole di Cornelio, precisando in modo generico di aver attinto ad altri autori, pur con autonomia di giudizio (Dolce, 1565a, c. 19v). Svincolandosi dallo schema di Pellegrino Morato, infatti, il quale traeva l'occasione del dialogo da un proprio sonetto sul tema cromatico commentandolo verso per verso, ${ }^{27}$ Dolce esamina i significati dei vari colori, ritagliandosi (anche sulla scorta di riferimenti a brani letterari diversi) spazi personali di dissenso o autonoma riflessione. Egli dialoga a distanza, pur senza citarlo, con Morato, da un lato ricalcando il suo dettato, dall'altro introducendo prospettive diverse: ad esempio il poligrafo aggiunge all'interpretazione metaforica del verde come perdita delle energie vitali - metafora peraltro corroborata dal rinvio a una propria terzina in stile bernesco ("Amanti, la candela è giunta al verde | [...]") - l'equivalenza di verde e speranza. ${ }^{28} \mathrm{E}$ ancora, se per Morato (1543, c. B3r) "il rosso ha poca sicurezza", come recita il secondo verso del suo sonetto, Dolce (1565a, 22v) a tale assunto affianca il significato di vendetta evocato dal colore del sangue. E così, secondo un simile impianto, vengono esaminati tutti i colori del sonetto di Morato.

Morato concludeva il trattato con la convinzione di aver fornito spunti a chi volesse "a qualche bello modo isprimere il suo concetto" (Morato, 1543, c. D3v). Per Dolce la sezione sul significato dei colori doveva preludere ad altro, ovvero alla parte del dialogo relativa al valore simbolico degli oggetti offerti in dono a potenziali destinatari. E infatti, come accennato sopra, la sezione conclusiva del trattato di Morato, sulla varietà e l'accostamento dei colori, con una sorta di tecnica a incastro coincide, anche sotto il profilo testuale, con la parte del dialogo di Dolce introduttiva al discorso sui doni. Sono anzitutto chiamati in causa alcuni problemi di fondo nello studio dei colori (con riferimento alla trattatistica antica, greco-latina), ovvero la questione del loro carattere accidentale, che (secondo Morato / Dolce) non deve inficiare la percezione della varietà cromatica, né le classificazioni convenzionali dell'uso moderno. Sulla scorta di Morato (sempre con minimi strategici aggiustamenti formali rispetto al suo dettato), Dolce ribadisce del resto la soggettività della percezione degli effetti coloristici, indicando il sottile confine tra codici etici ed estetici, tra gusto personale e canoni di convenienza e decoro. Nell'ambito di argomentazioni pressocché identiche anche sul piano testuale (cfr. Morato, 1543,

26. Già Salza (1901, p. 320-322) aveva segnalato taciti recuperi, nonché casi di errata ricezione del dettato del modello, pur riconoscendo l'interesse del trattato, soprattutto per le citazioni in un contesto originale di testi della tradizione italiana. I numerosi passi ripresi da Dolce "alla lettera" o "quasi alla lettera" risultano evidenziati da Paola Barocchi (1971-1977, vol. II, pp. 2159-2178). Diverso è il peso dato a tali coincidenze testuali da Terpening, R.H. (1997, p. 159 e nota 51), che considera la diversa consistenza delle due opere.

27. Per la tradizione dei sonetti sui colori: Barocchi, P. (1971-1977), vol. II, p. 2159.

28. Cfr. Morato. F.P. (1543), c. A3v, B1r-B3r; Dolce, L. (1565a), cc. 19v-22v. 
cc. D2v/Dolce, 1565a, 35v-36v) Dolce innesta un riferimento alle sopravesti ariostesche, esempio di un'ecfrasi che riporta al costume cavalleresco e all'araldica, poiché divise e insegne identificano i cavalieri. ${ }^{29}$ Le considerazioni di entrambi sul vestiario includono, peraltro, come fulcro del ragionamento il sintagma di un versetto biblico (circum amicta varietate: Ps. 44, 10,14), la cui controversa interpretazione appare banalizzata e ricondotta alla concretezza di abitudini quotidiane, ovvero alla corrispondenza tra tipo di abiti e parti del corpo.$^{30}$ Le riflessioni di Morato, mirate a criticare il costume di chi indossa abiti di foggia straniera sulla scorta di Plauto e Luciano, ${ }^{31}$ vengono inoltre amplificate da Dolce con un richiamo all'invettiva dantesca contro le "sfacciate donne fiorentine" (Pg. XXIII, 97-105), rendendo ancora più complessi i percorsi della memoria e le associazioni analogiche. ${ }^{32}$

Nell'ultima parte del trattato di Dolce, in cui prende forma una analisi di potenziali doni, si associano colori, odori, sapori, erbe aromatiche e frutti, ma anche oggetti e libri, cui corrispondono altrettanti impliciti messaggi simbolici per i destinatari di tali doni.

La Tavola finale ("Tavola de' colori") distingue gli oggetti in tre gruppi, in tutti i casi con i lemmi incolonnati e affiancati dal numero di carta corrispondente: al primo indice, relativo ai colori (c. $87 \mathrm{rv}$, infra, fig. 11 e 12), segue quello dei "Donativi diversi" ("quello che significano") per i quali l'ordinamento alfabetico coincide con la sequenza della trattazione (ivi, c. $87 v$ e infra, fig. 12); l'ultimo gruppo è indicizzato nella Tavola senza un ordine alfabetico, secondo lo sviluppo delle argomentazioni (ivi e infra, fig. 12 e 13) e non senza omissioni rispetto agli oggetti effettivamente considerati. ${ }^{33}$ Il punto di riferimento è costituito dalla sezione finale del trattato di Morato, che consiste in una semplice tavola alfabetica, una sorta di lemmario corredato di definizioni, il

29. Sulle “sopraveste discritte dall'Ariosto" (Dolce, L.,1565a, 36v): Salza, A. (1901), pp. 333-62.

30. Per i testi (Morato, F.P., 1543, c. D3r e Dolce, L., 1565a, 36v), infra, fig. 10a e 10b. Sul versetto del salmo: Pavan, V. (1988).

31. Morato, F.P. (1543), D3r (vd. fig. 10 e cfr. 10b). Si noti come, nel riferimento a Plauto e Luciano, ripreso alla lettera da Dolce, si incrocia il concetto di "peregrinus" ('forestiero') - visto nella sua accezione 'negativa', deducibile da Pseudolus, 964 e dal profilo del multiforme protagonista di Luciano (in De morte Peregrini) - con quello di "uccello", con cui, in Poenulus, 975, è spregiativamente qualificato il cartaginese per il suo modo di vestire. Si veda comunque per questo passo il commento di Osborne (2019), p. 420, nota 716.

32. Una precisazione va fatta per l'errore tipografico presente in Dolce, L. (1565a), $37 r$ (vd. fig. 10b), uno dei tanti di cui l'editore stesso avvertiva i lettori, facendo leva sulla normalità degli errori di stampa e sul fatto che l'autore non avesse "potuto attendere alla correttione con quella diligenza che bisognato sarebbe" (Dolce, L., 1565a, $4 v$ e cfr. anche l'avvertenza in calce all'errata corrige, c. [88] v, infra, fig. 14). Infatti errori di tal genere non solo costituiscono indizi di un modo di lavorare affrettato, sotto le pressioni del mercato editoriale, ma devono suggerire le dinamiche redazionali tipiche di opere che rimaneggiano altri testi, dove ampie coincidenze testuali, scarti e fraintendimenti appaiono inquadrabili in una fenomenologia della copia, con tutte le possibili conseguenze metodologiche nel caso di un approccio ecdotico al Dialogo dolciano.

33. Pure tali omissioni sono indicative di dinamiche redazionali tutte da indagare, soprattutto se si considera il finale disordine argomentativo (cfr. infra). 
cui titolo esplicita il criterio utilizzato per desumere i significati (Morato, 1543, c. D4r, infra, figg. 15a e 15b). Basta confrontare la prima carta della Tavola di Morato con l'inizio della sezione sui doni del dialogo dolciano per comprendere la tecnica con la quale Dolce innesta nel contesto dialogico del trattato gli enunciati schematici del suo antigrafo, innervandoli di utili precisazioni, spesso accompagnate anche da citazioni di versi. Comparando sistematicamente il dettato dei due trattati, si apprezzano le sottili differenze di intenti e funzioni che le secche definizioni acquistano, calate nella nuova cornice. E soprattutto occorre sottolineare come, uscendo dallo stretto ambito del mondo animale e vegetale o degli stessi oggetti contemplati da Morato, Dolce si distacchi dal suo modello, per procedere con una trattazione indipendente e originale: ad esempio quando passa in rassegna i significati allegorici di figure mitologiche e divinità pagane, talora con il parallelo riferimento a poesie e dipinti. $\grave{E}$ il caso del satiro, simbolo di lascivia, su cui Dolce, per voce di Cornelio, si sofferma con una libera resa in prosa volgare di un carme latino di Bembo (Carmina, 3: "Faunus ad Nymphas": Dolce, 1565a, c. 51rv), citando anche un paesaggio di Tiziano con una "Ninfa che vi siede insediata da due satiri" e ricordando insieme tutte le relative raffigurazioni poetiche dell'Arcadia di Sannazaro (ivi, c. $51 \mathrm{rv}$ ). E ancora, per il mito di Apollo e Marsia, allegoria della temerarietà, viene richiamata "una pittura di Antonio da Correggio" che Dolce (Cornelio) dichiara di aver visto "giorni addietro" (ivi, c. $51 v$ ). E anche questo è un passo di particolare interesse, su cui potrebbe esercitarsi la critica per ricostruire i percorsi eruditi e storico-culturali, tra ambiti poetici e artistici. ${ }^{34}$

Le domande semplici, insistenti e apparentemente ingenue di Mario nel Dialogo dolciano aprono di certo un mondo di sostanze naturali, animali e oggetti (preziosi o banali e umili), tutti portatori di significati nascosti, come del resto avveniva per il Dialogo dell'imprese militari e amorose di Giovio e per il Dialogo della memoria dello stesso Dolce. Si tratta del mondo virtuale degli emblemi, delle imprese e delle marche editoriali, ${ }^{35}$ quest' ultimo familiare a Dolce, il quale, come revisore, collaborò con stamperie prestigiose (tra le quali quella dei Giolito).

34. Si può allo stato attuale osservare che Dolce fornisce qui testimonianza di un perduto dipinto di Tiziano (non è registrato da Cagli, C. \& Valcanover, F., 1969), ma riprodotto in una stampa seicentesca realizzata da Giovan Francesco Grimaldi: Bartsch, A. (1819), p. 109, n. 44. La stampa si conserva a Milano, Castello Sforzesco, Civiche raccolte grafiche e fotografiche: scheda SIRBeC: http://www.lombardiabeniculturali.it/stampe/schede-complete/ H0110-07576/. Per l'“Apollo e Marsia” si può escludere invece la paternità di Antonio da Correggio (cfr. Bevilacqua, A. \& Quintavalle, A.C., 1970, p. 111, scheda 91). Dolce doveva aver visto in realtà il dipinto di Bronzino (o una sua copia) sul cui modello Giulio Sanuto eseguì nel 1562 una incisione in tre fogli su rame, ora conservata a San Francisco, Museum of Fine Arts. L'attribuzione a Correggio si deve proprio a Giulio Sanuto (il quale dichiarò di aver tratto l'immagine "ex clarissimi pictoris Antonii de Correggio pictura"). Sull'incisione di Sanuto: Mataloni, C. (on line), senza data, scheda 56; per il dipinto di Bronzino: Mataloni, C., (on line), senza data, scheda 43.

35. Come osserva anche Torre, A. (2001), p. XXXVIII. 
In risposta al quesito di Mario sul significato simbolico della biscia, Cornelio indica ad esempio la marca tipografica di Francesco Rosso, che, accompagnata dal motto Dilexisti malitiam super benignitatem, fu adottata nella terza edizione ferrarese del Furioso per indicare la malignità dei detrattori dell'opera (fig. 1); ad essa si associa il ricordo della xilografia introdotta da Mazocco del Bondeno in apertura della prima edizione del Furioso (Ariosto, 1516) con lo sciame d'api e il motto Pro bono malum (fig. 2). ${ }^{36}$ Interessante anche il richiamo alla marca tipografica di Aldo Manuzio (fig. 3), che scaturisce dalla spiegazione congiunta del significato del delfino ("pesce velocissimo" che indica "prestezza") e dell'ancora, simbolo di "fermezza". ${ }^{37}$

Discutendo sul dono di una "gatta", che nelle case procura danno, ma nello stesso tempo è utile a scacciare i topi, Cornelio sottolinea il senso dell'insegna tipografica di Marchiò (Melchiorre) Sessa, la gatta con il topo in bocca, con il motto Dissimilium infida societas, che allude alla poco duratura amicizia tra esseri dissimili. ${ }^{38}$

Non poteva mancare il richiamo all'insegna dei Giolito, con la fenice (simbolo di rinascita) e il motto, nelle sue varianti in latino e in volgare (Semper eadem, De la mia morte eterna vita i' vivo, Vivo morte refecta mea), che appariva

36. Cfr. Dolce, L. (1565a), c. $50 \mathrm{rv}$. Sulle "imprese" ariostesche e i relativi motti cfr. almeno Casadei, A. (1997), pp. 150-151; Masi, G. (2002); Ferretti, F. (2012), p. 109; Forni, G. (2012, soprattutto le pp. 94-96); Petrone, M. (2014), pp. 249-256. Sembra che si possa cogliere, però, una sottile differenza tra la biscia, allusiva alla malignità, e il serpente, indicato subito dopo (Dolce, L., 1565a, c. 50v) quale simbolo di prudenza sulla scorta di un passo evangelico (Mt. 10 16). Il serpente, infatti, rinnova la propria pelle, "alludendo quasi alla immortalità dell'anima" (Dolce, L., 1565a, c. $50 v$, con un percorso di rinvii e citazioni che vanno da Eneide, II, 471-473 a Orlando furioso, XVII, 11, 2-6, da Catullo, V, 4-6 a Sannazaro, Arcadia, XI, Poi che 'l soave stile e 'l dolce canto, vv. 61-63). Sulla simbologia del serpente nel Furioso cfr. almeno Stroppa, S. (2006), Petrone, M. (2014), pp. 198-259, Brancati, F. (2016), anche per ulteriori rinvii bibliografici.

37. Dolce, L. (1565a), c. 54r. Dolce attribuisce l'impresa e il motto "Festina lente" a Tiberio, contrariamente alla tradizione che rimanda ad Augusto (cfr. Svetonio, De vita Caesarum, 25, Aulo Gellio, Noctes Atticae, 10, 11 e Macrobio, Saturnalia, 6, 8, 9, e cfr. Erasmo, Adagia, 1001). Il motto aldino apparve per la prima volta in lingua greca nella premessa a Poliziano, A. (1498), c. al $v$; venne poi affiancato dalla traduzione (nella forma Semper festina tarde) nell'Hypnerotomachia Poliphili nella didascalia in calce all'immagine dell'ancora e del delfino (Colonna F., 1499, c. d[7]; 1998, t. I, p. 69: fig. 4). Giovio, P. (1555) lo riporta nella variante Propera tarde (cfr. Giovio, P., 1978, p. 35), mentre la forma Festina lente, con attribuzione ad Augusto, è accolta da Ruscelli nell'edizione a sua cura del Dialogo gioviano (Giovio, P., 1556, c. 4). Cfr. Ordine, N. (2014); Bongiovanni, M.B. (2015); Vacalebre, N., 2016, p. 151 e soprattutto Pagliaroli, S. (2019). Una breve digressione sull'impresa e sul motto utilizzati da Aldo si registra anche in Dolce (1565a), c. 69r, a proposito del significato simbolico degli "sproni", donati per scuotere dalla pigrizia.

38. Dolce, L. (1565a), c. $56 v$ e fig. 5 , che riproduce una delle dodici varianti dell'impresa, sulle quali Curi Nicolardi, S. (2019), pp. 308-312; allo stesso volume si rinvia per la storia dei Sessa e per la relativa bibliografia, e ad indicem per la collaborazione di Dolce in qualità di revisore editoriale. Ė assente invece nel Dialogo il richiamo all'altra marca editoriale utilizzata da Melchiorre Sessa dal 1558 in poi, ovvero la figura di Pegaso in volo, che peraltro compare nel frontespizio di alcune opere dello stesso poligrafo veneziano (tra cui il Dialogo della memoria e il Dialogo dei colori: Dolce, L., 1562 e 1565a; vd. fig. 8). 
a Dolce particolarmente appropriato a valorizzare il ruolo degli editori, capaci di rendere immortali gli scrittori mediante la diffusione delle loro opere. ${ }^{39}$

Del resto, almeno una cinquantina degli elementi presi in considerazione da Dolce (e citati nella Tavola) si ritrova presente, singolarmente o in combinazioni varie, in marche tipografiche di editori già attivi o di epoca successiva. Pertanto si conferma la funzionalità dell'opera nell'offerta di un modello di costruzione delle rappresentazioni iconiche e delle chiavi per le loro interpretazioni.

Al libro, in quanto oggetto (osservato quale contenitore, oltre che come latore di messaggi e contenuti) e simbolo di "ricovramento di libertà o di persona libera", ${ }^{40}$ Dolce dedica interessanti pagine, in aggiunta alle osservazioni (tradotte alla lettera da Telesio, nella prima parte sui colori) relative a un antico uso di distinguere l'Odissea e l'Iliade mediante i diversi colori delle legature: per la prima il ceruleo, che evoca la navigazione di Ulisse, per la seconda il "sanguigno", che evoca le battaglie. ${ }^{41}$ Questa considerazione, mutuata dall'erudito cosentino, sugli aspetti 'esterni' del libro, si sviluppa nell'ultima parte dell'opera, dove i testi degli autori si materializzano in altrettanti oggetti da donare, ciascuno con la propria funzione e utilità, come avviene per un "Petrarca", simbolo di amore "spirituale" (Dolce, 1565a, cc. $71 v-72 v$ ); e ancora: per un "Dante", veicolo di insegnamenti morali (ivi, cc. 72v-73v); per un "Virgilio", da destinarsi a persona "eccellentissima" (ivi, c. 73v); per un "Furioso", idoneo per la varietà dei suoi contenuti a trasmettere messaggi molteplici, etici e civili (ivi, c. 73v); per le sacre scritture, basilari per l'insegnamento religioso, ma da leggersi esclusivamente nella traduzione di S. Girolamo (ivi, c. $74 \mathrm{rv}) ;{ }^{42}$ per gli Asolani di Bembo, che illustrano la teoria platonica dell'amore (ivi, cc. $74 v$ 75r); per l'Arcadia di Sannazaro, che mediante la descrizione della vita rustica suggerisce l'immagine della primitiva età felice (ivi, c. $75 \mathrm{rv}$ ). ${ }^{43}$ Questa prima

39. Dolce, L. (1565a), cc. 56r-57v. Per tale simbologia Dolce cita versi di Petrarca (Rvf, CLXXXV, 1-4 e XXXI, 9-15) e di Bembo (CLV, 1-4). Ludovico Dolce ebbe un ruolo fondamentale all'interno della tipografia, quale collaboratore di Giolito, a partire dal 1542. A sua cura è la monumentale edizione del Furioso giolitino del 1542 (che inaugurava una fortunata serie di edizioni con ricchi apparati illustrativi oltre che esegetici), nel cui frontespizio istoriato, lungo il contorno dell'ovale che contiene l'insegna della fenice, si legge il motto "Vivo morte refecta mea". Cfr. Nuovo, A. - Coppens, C. (2005), in particolare pp. 22-25 e passim per varianti dell'insegna (su cui cfr. Edit 16; e si vedano qui le figure 6 e 7). Su Dolce curatore di edizioni del Furioso e anzi iniziatore della critica ariostesca con la stampa veneziana di Pasini e Bindoni del 1535: Villari, S. (2013); Lo Rito, C. (2013); Iorio, G. (2013).

40. Dolce, L. (1565a), c. $42 v$. Cfr. Morato, F.P. (1543, c. D[6]r): "Libro, recuperatione della persona libera".

41. Dolce, L. (1565a), c. 9r; Telesio, A. (1528), c. a3v. Cfr. Terpening, R.H. (1997), p. 160.

42. Esplicita la polemica con Erasmo da Rotterdam (Novum instrumentum, Basilea, Froben, 1516) per il suo distacco dalla vulgata di S. Girolamo.

43. Dolce, peraltro, riconduce il concetto di età felice ai versi di Boezio (De consolatione philosophiae, II, 5, 1-2: "Foelix nimium prior aetas / Contenta fidelibus arvis"), in luogo di richiami più scontati, come, ad esempio, a Dante, Pg., XXII, 148-150 ("Lo secol primo, quant'oro fu bello [...]”), di cui Boezio è un probabile precedente. Il rinvio a Boezio non era 
serie di libri si interrompe con Giovenale, indicato come utile per l'efficacia morale del genere satirico, praticato anche da Ariosto (Dolce, 1565a, cc. 75v$76 r$ ) e con Plinio, destinato a persone bisognose di insegnamento o dotate di acuto ingegno (ivi, c. 76 r). ${ }^{44}$

In questo excursus sui libri, in cui si ritrovano i best seller del tempo, che circolavano nel mercato editoriale anche con le cure di Dolce, ${ }^{45}$ un grande assente tra i classici in volgare è il Decameron, nonostante fosse stato oggetto delle attenzioni critiche, linguistiche, e anche 'filologiche' del poligrafo veneziano (si pensi alle edizioni giolitine del 1546 e del 1552). Ma per spiegare questa assenza dovrebbe essere sufficiente osservare come i libri elencati da Dolce nel suo Dialogo siano tutti indicati come strumenti di elevazione etica o di conoscenza, mentre sul Decameron (accolto da Bembo in poi come modello linguistico per la prosa, seppure con qualche riserva) gravavano all'epoca pregiudizi morali per i caratteri 'licenziosi' di molte novelle.

Una breve disamina di oggetti (gli occhiali, l'asciugatoio, il coltello, lo specchio) introduce una cesura nella rassegna di libri, e a proposito dello specchio Dolce si cimenta nella lettura, a suo dire senza precedenti, di RvfCCCLXI (Dicemi spesso il mio fidato speglio). ${ }^{46}$

È a quel punto che si riparte con i libri, e in particolare con i classici latini, la cui posizione all'interno di un canone è sempre precisata anche in modo problematico, seguendo gli schemi del tempo: lo storico Livio, anzitutto, è inquadrato per le sue qualità storiografiche e stilistiche, osservato nei suoi rapporti con Sallustio e Cesare, e indicato pragmaticamente come testo utile per i professionisti delle armi, grazie alle sue piene descrizioni di battaglie (Dolce, 1565a, c. 78v); Lucano e Stazio offrono un esempio tutto in negativo, attestando la diffidenza tipica dei classicisti nei confronti degli autori dell'età argentea, e pertanto donare la Farsalia o l'Achilleide acquista il senso di un suggerimento a evitare l'imitazione di quello stile "gonfio", da cui ad esempio si astenne Ariosto, imitando accortamente Virgilio (Dolce, 1565a, cc. 78v-79v). Diverso è il caso delle Heroides ovidiane, opera "buonissima [...], purissima,

facile o immediato, come conferma il fatto che Alessandro Vellutello nel proprio commento (Comedia" di Dante Aligieri con la nova esposizione, Venezia, Marcolini, 1544) attribuiva i versi boeziani a Virgilio. Per questa informazione: Pirovano, D. (2007), p. 120.

44. In proposito Dolce apre una breve digressione sul giudizio petrarchesco su Plinio ("Quel Plinio Veronese suo vicino | a scriver molto, a morir poco accorto": Tr. Fam, III, 44-45), apprezzando il rilievo sulla morte causata da imprudenza, ma sorprendendosi nel contempo dell'attribuzione di un'origine veronese piuttosto che comasca. Ma non è questa la sede per indagare sul percorso di Dolce in questa tradizione esegetica. Per i versi petrarcheschi: Petrarca, F. (1996), p. 446-447.

45. Per un elenco delle opere curate da Dolce: Terpening, R. H. (1998), pp. 266-269.

46. Dolce, L. (1565a), cc. 76r-78v. Lapproccio di Dolce a questo sonetto apre qualche interessante pista di indagine, sul versante della storia dell'esegesi petrarchesca e della tradizione testuale, anche per la polemica che Dolce introduce rispetto alla resa testuale (paragrafematica, diremmo oggi) di RvfCCCLXI, 5-6. Il sonetto è commentato, peraltro nelle edizioni a cura di Giovanni Andrea Gesualdo (1553), c. 338rv e di Alessandro Vellutello (1560), c. $130 r$. 
latinissima e piena di amoroso affetto", perfetto dono per chi "fosse scrittore di cose amorose, latine o volgari" (Dolce, 1565a, c. 79r), e ancora degli elegiaci latini (Catullo, Tibullo, Properzio), ma anche del perduto Gallo, di cui Dolce metteva in dubbio, mediante la voce di Cornelio, l'attribuzione dei frammenti contenuti nella raccolta veneziana del 1549 (ivi, cc. 79v-80r). ${ }^{47}$ Per valorizzare l'apporto degli elegiaci latini, tracciando un filo diretto con la tradizione lirica in volgare, Dolce citava le Stanze di Bembo (le ottave 21 e 22, nelle quali al ricordo di Catullo, Properzio, Tibullo, Gallo, segue quello di Cino da Pistoia, Dante e Petrarca: Dolce, 1565 a, c. $80 \mathrm{rv}$ ). Viene poi proposta una serie di libri "dozzinali" o "goffi" da destinarsi a persone mediocri, come erano considerate le rime di Serafino Aquilano, di Antonio Tebaldeo, di Vincenzo Calmeta. La citazione dell' incipit di un componimento attribuito a quest' ultimo ${ }^{48}$ dà modo a Dolce di soffermarsi sul genere della frottola (o della predica amorosa) e su alcune rime extravaganti petrarchesche (Dolce, 1565a, cc. $80 v-81 v$ ).

$\mathrm{Al}$ di là di una valorizzazione simbolica dell'oggetto-libro, le valutazioni sulle opere, scandite da puntualizzazioni relative a stili, generi e canoni, consentono di intravedere i fili di un dibattito sulle tendenze della produzione antica e moderna e di entrare nel vivo della cultura coeva.

Verso la fine del volume, quando si ritorna a una disamina di oggetti, nel dialogo tra Mario e Cornelio si registra un affastellarsi disordinato di domande, sottolineato dallo stesso Cornelio quando viene chiamato a pronunciarsi sulla testa di un Laocoonte, subito dopo aver parlato di cose di tutt'altro genere, come "frumento, avena e loglio" "Tu non serbi ordine alcuno in queste tue domande", ivi, c. 84r). In modo del tutto casuale, inoltre, alla fine del Dialogo Mario chiede a Cornelio di spiegare e tradurre dei versi latini, come l'epigramma di un antico Plucice o i versi dedicati al racconto della vicenda di Omonea e Achimeto. ${ }^{49}$ E proprio qui si conclude il trattato (ivi, c. $86 v$ ), seguito dalle Tavole dei colori e dei doni di cui si è parlato sopra.

L'interruzione del discorso, privo di una effettiva conclusione, lascia l'impressione di un'opera intesa come un contenitore elastico, una struttura enciclopedica potenzialmente aperta e suscettibile di aggiunte e inserzioni, come se l'autore si fosse fermato per ragioni di tempo e di opportunità, ma non per aver esaurito la rete simbolica delle infinite analogie tra oggetti e significati.

47. Catullus, Tibullus, Propertius. His accesserunt Corn. Galli fragmenta, Venetiis, apud Hieronimum Scotum (la biografia e le elegie attribuite a Cornelio Gallo si leggono alle cc. $132 r-143 r)$.

48. "Omnia vincit amor [...]". Dolce poteva leggerlo in una raccolta cinquecentesca di lirica cortigiana: Compendio di cose nuove di Vincenzo Calmeta e altri auctori. Venezia: Zoppino, 1507. Su questa suggestiva attribuzione a Calmeta e sulla qualifica di "predica" che Dolce appone a tale componimento si sofferma Largaiolli, M. (2008), pp. 66-67 (dove è riportato un significativo stralcio del Dialogo dolciano).

49. L'epigramma è da Dolce citato in latino, ma si trova tradotto in volgare, con attribuzione a Plucice, nella raccolta di Claudio Tolomei (1539), c. N[3] $v$. I versi latini sulla vicenda di Omonea e Achimeto, si potevano leggere, ad esempio, nel Dictionarium di Ambrogio Calepino, s.v. Homonea (ho consultato l'ed. di Venezia, Ioan. Gryphius, 1550). Si rinvia ad altra sede una adeguata indagine sulla tradizione di questi testi. 
E anche la mia analisi può fermarsi qui, con la consapevolezza di aver posto soltanto qualche tessera per la ricostruzione dei frastagliati percorsi della comunicazione iconica di quell'ultimo scorcio del XVI secolo; un tipo di comunicazione che ha contribuito a gettare le basi della cultura e della poetica barocca. E non a caso Emanuele Tesauro, nel fornire nel suo Cannocchiale aristotelico una compiuta codificazione dell'arte dell'emblematica, si riallacciava significativamente alla speculazione umanistica:

[...] per emblema propiamente si intende hoggidì dagli Humanisti [...] un simbolo populare, composto di Figura, e parole, significante per modo di Argomento alcun Documento appartenente alla vita humana, et perciò esposto per fregio, et ornamento ne' Quadri, nelle Sale, negli Apparati, nelle Academie, overo impresso né libri con Imagini, e spiegationi per publico insegnamento del popolo. Dove per populare et per popolo tu non devi'ntendere la ignara Plebe, ma quegli mezzani ingegni, che pure intendono il latino, et delle lettere humane sono mediocremente infarinati $\left[\ldots . . . .^{50}\right.$

Sembra proprio che si possa trovare qui una chiave di lettura anche del Dialogo dei colori di Dolce, la cui destinazione coincide perfettamente con il profilo che Tesauro delinea del pubblico "populare".

\section{Bibliografia}

Ariosto, L. (1516). Orlando furioso de Ludouico Ariosto da Ferrara. Ferrara: Giouanni Mazocco dal Bondeno.

Ariosto, L. (1532). Orlando furioso di messer Ludouico Ariosto nobile ferrarese nuouamente da lui proprio corretto e d'altri canti nuoui ampliato con gratie e priuilegii. Ferrara: Francesco Rosso da Valenza.

Ariosto, L. (2006). Orlando furioso secondo la princeps del 1516 (edizione critica a cura di M. Dorigatti, con la collaborazione di G. Stimato. Firenze: Olschki.

Arroyo Esteban, S., (2015). Pietro Aretino, Lodovico Dolce y el "Juicio Final" de Miguel Ángel, Studia Aurea, 9, 339-360.

Arroyo Esteban, S. (2016). El "Dialogo della pittura" antes del "Dialogo della pittura". Lodovico Dolce entre pintura y poesía. In Marini, P. \& Procaccioli, P. (edd.) (2016), pp. 465-504.

Bartsch, A. (1819), Le peintre graveur, vol. XIX. Vienna: Pierre Mechetti.

Barocchi, P. (ed.) (1971-1977). Scritti d'arte del Cinquecento. Bari / Milano-Napoli: Laterza / Ricciardi.

Bevilacqua, A. \& Quintavalle, A.C. (1970). L'opera completa del Correggio. Milano: Rizzoli.

Bolzoni, L. (1995). La stanza della memoria. Modelli letterari e inonografici nell'età della stampa. Torino: Einaudi.

Bongiovanni, M.B. (2015). "Festina lente": due incisioni dell" "Hypnerotomachia Poliphili” e la marca tipografica di Aldo Manuzio, Bollettino telematico dell'arte, 771 (10 maggio) http://www.bta.it/txt/a0/07/bta00771.html

50. Tesauro, E., (1663), pp. 645-646 (il corsivo è nella stampa). Rinvio in proposito anche alle illuminanti pagine di Ezio Raimondi dedicate al barocco ("Lo specchio del barocco e le immagini del presente”, in Raimondi, E., 1995, pp. 3-19). 
Borghini, R. (I 584). Il riposo, in cui della Pitture e della Scultura si favella, de' più illustri Pittori e Scultori e delle piu famose opere loro si fa menzione e le cose principali appartenenti a dette arti sinsegnano. Firenze: Giorgio Marescotti.

Brancati, F. (2016). "In serpentile scorza": presenze serpentine nel "Furioso", Carte Romanze, 4/2, 175-205 http://riviste.unimi.it/index.php/carteromanze/index

Cagli, C. \& Valcanover, F. (1969). L'opera completa di Tiziano. Milano: Rizzoli.

Casadei, A (1997). Il "Pro bono malum» ariostesco e la Bibbia. In Id., La fine degli incanti. Vicende del poema cavalleresco nel Rinascimento. Milano: FrancoAngeli, pp. 149-151.

Cian, V. (1894). Del significato dei colori e dei fiori nel Rinascimento italiano. Torino: Roux (estr. da Gazzetta letteraria, 13-14).

Ciccarese, M.P. (2016). "Scarabaeus clamans": la costruzione di una simbologia, Vetera Christianorum, 53, 77-98.

Colonna, F. (1499). Hypnerotomachia Poliphili. Venezia: Aldo Manuzio [vd. ristampa anastatica: Colonna, F. (1998), t. I].

Colonna, F. (1980). Hypnerotomachia Poliphili (Edizione critica e commento a cura di G. Pozzi e L. A. Ciapponi). Padova: Editrice Antenore.

Colonna, F. (1998). Hypnerotomachia Poliphili (a cura di M. Ariani e M. Gabriele). T. I: riproduzione dell'edizione aldina del 1499; t. II: introduzione, traduzione e commento. Milano: Adelphi.

Collin, F. (2011), recensione a Antonio Telesio de Cosenza, Petit traité des couleurs latines (De coloribus libellus), éd. M. Indergand et C. Viglino, Cahiers de recherches médiévales et humanistes [en ligne]: http://journals.openedition.org/crm/12315.

Curi Nicolardi, S. (2019), Melchiorre Sessa tipografo ed editore (Venezia 1506-1555).

Milano-Udine: Mimesis Editore.

Doglio, M.L. (1978). Introduzione a Giovio P. (1978), pp. 9-27.

Dolce, L. (1545). Dialogo della institution delle donne. Secondo li tre stati che cadono nella vita humana. Venezia: Gabriel Giolito de Ferrari.

Dolce, L. (1550). Osservationi nella volgar lingua di m. Lodovico Dolce. Divise in quattro libri. Venezia: Giolito del Ferrari e fratelli.

Dolce, L. (1557). Dialogo della pittura, intitolato l'Aretino. Nel quale si ragiona della dignità di essa pittura, e di tutte le parti necessarie, che a perfetto pittore si acconvegono: con esempi di pittori antichi et moderni: e nel fine si fa metione della virtù e delle opere del divin Titiano. Venezia: Gabriel Giolito de Ferrari.

Dolce, L., (1562). Dialogo nel quale si ragiona del modo di accrescere e conservar la memoria. Venezia: Gio. Battista e Marchiò Sessa.

Dolce, L. (1565a). Dialogo di M. Lodovico Dolce nel quale si ragiona delle qualità, diversità e proprietà dei colori. Venezia: Gio Battista, Marchio Sessa, et fratelli. [vd. anche la trascrizione, a cura di C. Ruggeri e A. Michelis: https://www.liberliber.it/ mediateca/libri/d/dolce/dialogo_dei_colori/pdf/dialog_p.pdf).

Dolce, L. (1565b). Libri tre di m. Lodovico Dolce, nei quali si tratta delle diverse sorti delle gemme che produce la natura, della qualità, grandezza, bellezza e virtù loro. Venezia: Giovan Battista, Marchio Sessa et fratelli.

Dolce, L. [1565c]. Somma della Filosofia d'Aristotele, e prima della Dialettica. Raccolta da m. Lodouico Dolce. Venezia: Gio Battista, Marchio Sessa, et fratelli.

Dolce, L. (1913), Dialogo dei colori (ed. D. Ciàmpoli). Lanciano: Carrabba.

Dolce, L. (1968). Dialogo della pittura. In M. W. Roskill, Dolce's "Aretino" and Venetian Art Theory of the Cinquecento, pp. 84-195 [testo italiano / inglese]. New York: New York University Press, 1968. 
Dolce, L. (1996). Dialogue de la peinture intitulé l'Arétin (ed. L. Fallay d'Este, trad. N. Bauer). Parigi: Klincksieck.

Dolce, L. (2001). Dialogo del modo di accrescere e conservar la memoria (ed. A. Torre). Pisa: Edizioni della Normale.

Dolce, L. (2004). I quattro libri delle Osservationi (ed. a cura di P. Guidotti). Pescara: Libreria dell'Università.

Dolce, L. (2010). Diálogo de la pintura, titulado Aretino, y otros escritos de arte (edición de S. Arroyo Esteban. Prologo de F. Checa Cremades). Madrid: Ediciones Akal.

Dolce, L. (2014). Diciamolo con i fiori (e con altri doni). Dal "Dialogo dei colori" di Lodovico Dolce (a cura di L. Gasparotto), Engramma, 115, online. http://www. engramma.it/eOS/index.php?id_articolo=1554.

Dolce, L. (2015). Lettere (a cura di P. Procaccioli). Manziana (Roma): Vecchiarelli.

Dolce, L. (2015). Dialogo della instituzion delle donne secondo li tre stati che cadono nella vita umana (ed. H. Sanson). Cambridge, UK: Modern Humanities Research Association.

Erasmo (1980). Erasmo da Rotterdam. Adagia. Sei saggi politici in forma di proverbi a cura di S. Seidel Menchi. Torino: Einaudi.

Fahy, C. (1989). L“'Orlando furioso” del 1532. Profilo di una edizione: Milano: Vita e pensiero.

Ferretti, F., (2012). Menzogna e inganno nel "Furioso". Versants, 59, 85-109.

Forni, G, (2012). Ironia e illusione nel IV del Furioso. In Id., Risorgimento dell'ironia. Riso, persona e sapere nella tradizione letteraria italiana. Roma: Carocci, pp. 94-115.

Gabriele, M. (2021). Il Polifilo e i geroglifici nel Quattrocento, in Hieroglyphica. Cléopâtre et l'Égypte entre France et Italie à la Renaissance (sous la direction de R. Gorris Camos), pp. 39-58. Tours: Presses Universitaires François Rabelais.

Genovese, G. \& Torre, A., (2019) (a cura di). Letteratura e arti visive nel Rinascimento. Roma: Carocci.

Gesualdo, G.A. (1553). Il Petrarcha con la spositione di Giovanni Andrea Gesualdo. Venezia: Domenico Giglio.

Giovio, P. (1555). Dialogo dell'imprese militari et amorose. Roma: Antonio Barrè.

Giovio, P. (1556). Ragionamento di mons. Paolo Giovio sopra i motti et disegni d'arme e d'amore che communemente chiamano imprese. Con un discorso di Girolamo Ruscelli intorno allo stesso soggetto. Venezia: Giordano Ziletti.

Giovio, P. (1978). Paolo Giovio, Dialogo dell'imprese militari e amorose (a cura di M.L. Doglio). Roma: Bulzoni.

Girardi, M.T. (2005). La lezione su "Verdi panni, sanguigni, oscuri o persi" (RVF XXIX) di Benedetto Varchi Accademico infiammato, Aevum, 3, 677-718.

Iorio, G. (2013). "Inventio" e "dispositio" nelle allegorie delle edizioni Giolito (1542) e Valvassori (1553). In Le sorti d'Orlando. Illustrazioni e riscritture del "Furioso" (a cura di M. Rossi e D. Caracciolo), pp. 39-53. Lucca: Pacini Fazzi.

Largaiolli, M. (2008). La "predica d'amore". Note sulla parodia sacra tra Quattro e Cinquecento, Italique, 11, 53-89.

Lo Rito, C. (2013). Due contendenti nell'agone del "Furioso". Ludovico Dolce e Girolamo Ruscelli curatori del poema ariostesco. In Le sorti d'Orlando. Illustrazioni e riscritture del "Furioso" (a cura di M. Rossi e D. Caracciolo), pp. 75-86. Lucca: Pacini Fazzi.

Maggi, V. - Lombardi, B. (1550). In Aristotelis librum de Poetica communes explanationes; Madii vero in eundem librum propriae annotationes. Venezia: Valgrisi.

Marini, P. \& Procaccioli, P. (edd.) (2016). Per Lodovico Dolce. Miscellanea di studi. I. Passioni e competenze del letterato. Manziana (Roma): Vecchiarelli. 
Masi, G. (2002). I segni dell'ingratitudine. Ascendenze classiche e medioevali delle imprese ariostesche nel "Furioso", Albertiana, 5, 141-164.

Mataloni, C. (on line), senza data, 56. Apollo e Marsia, Iconos, scheda 56. http://www.iconos.it/le-metamorfosi-di-ovidio/libro-vi/apollo-e-marsia/ immagini/56-apollo-e-marsia/

Mataloni, C. (on line), senza data, 43. Apollo e Marsia, Iconos, scheda 43. http://www.iconos.it/le-metamorfosi-di-ovidio/libro-vi/apollo-e-marsia/ immagini/43-apollo-e-marsia/.

Morato, F.P. (1535). Del significato dé colori. Venezia: Giovan Antonio Nicolini da Sabio.

Morato, F.P. (1543). Del significato dé colori e de' mazzolli. Operetta di Fulvio Pellegrino Morato mantovano nuouamente ristampata. Venezia: Bartolomeo Imperatore \& Francesco Veneziano.

Nuovo, A. - Coppens, C. (2005). I Giolito e la stampa nell'Italia del XVI secolo. Genève: Droz.

Occolti, C. (1568). Trattato dé colori di Coronato Occolti da Canedolo. Nuovamente composto et stampato con l'aggiunta del significato di alcuni doni, dal medesimo data in luce. Parma: Seth Viotto.

Ordine, N. (2014). Introduzione. "Festina lente". Velocità e lentezza, folgorazione improvvisa e paziente ricerca nella scrittura. In Festina lente. Il tempo della scrittura nella letteratura del Cinquecento (a cura di C. Cassiani, M.C. Figorilli. Introduzione di N. Ordine). Roma: Edizioni di Storia e letteratura, pp. VII-XIV.

Osborne, R. (2019). Renaissance Colour Symbolism. Morrisville, North Carolina USA : Lulu Press, Inc.

Padoan, G. (1978). "Ut pictura poesis": le "pitture" di Ariosto, le "poesie" di Tiziano. In Id., Momenti del Rinascimento veneto, pp. 347-370. Padova: Editrice Antenore [anche in Tiziano e Venezia. Convegno internazionale di studi. Venezia, 1976, pp. 91-102. Vicenza: Neri Pozza, 1980].

Pagliaroli, S. (2019). Aldo Manuzio, Erasmo da Rotterdam, Michele Trivoli e il simbolo dell'ancora e del delfino. In Aldo Manuzio. Editore umanista e filologo (a cura di G. Comiati), pp. 151-186. Milano: Ledizioni.

Pavan, V. (1988). Girolamo e l'interpretazione antica di "circumdata varietate" (Sal. 44,10.14): la diversità dei carismi, Annali di Storia dell'Esegesi, 5, 239-252.

Petrarca, F. (1996), Trionfi, Rime estravaganti, codice degli abbozzi (ed. a cura di V. Pacca \& L. Paolino. Milano: Mondadori.

Petrone, M. (2014), Per un bestiario dell'Orlando furioso. Tesi di dottorato. Università della Calabria (supervisore Nuccio Ordine): http://dspace.unical.it:8080/jspui/ handle/10955/921

Pirovano, D. (2007), Alessandro Vellutello esegeta e filologo della "Commedia”, Rivista di studi danteschi, 7, 104-140.

Poliziano, A. (1498). Omnia opera. Venezia: Aldo Manuzio.

Pozzi, M. (1968). L"Ut pictura poësis" in un dialogo di L. Dolce. Giornale storico della letteratura italiana, 144 (446-447), 234-60.

Raimondi, E. (1995). Il colore eloquente. Letteratura e arte barocca. Bologna: Il Mulino.

Robortello, F. (1548). In librum Aristotelis de arte poetica explicationes. Firenze: Lorenzo Torrentino.

Salza, A. (1901). Imprese e divise d'arme e d'amore nell'“Orlando furioso" con notizia di alcuni trattati del ' 500 sui colori, Giornale storico della letteratura italiana, 38, 310-363. 
Sanson, H. (2015), Introduction to Dolce, L. (2015), pp. 1-75.

Saracco, L. (2012). Morato, Fulvio Pellegrino. In Dizionario biografico degli Italiani, vol. 76. Roma: Istituto della Enciclopedia italiana Treccani, pp. 539-540.

Sgarbi, M. (2015). Ludovico Dolce e la nascita della critica d'arte. Un momento della ricezione della poetica aristotelica nel Rinascimento, Rivista di estetica, 59, 163-82.

Stroppa, S. (2006). L'ira di Orlando. Orlando Furioso XLI 95-XLII 10, "Per leggere", $11,49-72$.

Telesio, A. (1528). Antonii Thylesii cosentini Libellus de coloribus, ubi multa leguntur praeter aliorum opinionem. Venezia: Bernardino Vitali.

Telesio, A. (2010). Petit traité des couleurs latines (De coloribus libellus) (éd. M. Indergand \& C. Viglino. Paris: Estienne.

Terpening, R.H (1997). Lodovico Dolce Renaissance man of letters. Toronto - Buffalo - London: University of Toronto Press Incorporated.

Tesauro, E. (1663). Il cannocchiale aristotelico, o sia Idea dell'arguta et ingeniosa elocutione, che serve a tutta l'arte oratoria, lapidaria, et simbolica. Esaminata co' principii del divino Aristotele. Seconda impressione, accresciuta dall'autore di due nuovi trattati, cioè, Dé concetti predicabili, et Degli emblemi. Venezia: Paolo Baglioni.

Tolomei, C. (1539). Versi, et regole de la nuova poesia toscana. Roma: Antonio Blado d'Asola.

Torre, A., 2001. Introduzione a Dolce, L. (2001), pp. I-LV.

Tramontana, A. (2021). Motivi di ecfrasi dantesca in Michelangelo secondo Benedetto Varchi: ancora sulle "Due Lezzioni” all'Accademia fiorentina, Quaderns

d'Italià, 26, pp. 81-96.

Vacalebre, N. (2016). "Festina lente". Un percorso virtuale tra le edizioni aldine della Biblioteca Trivulziana di Milano Università Cattolica. Milano: C.R.E.L.E.B - Università Cattolica / Milano: Edizioni CUSL. (anche on line: https://centridiricerca. unicatt.it/creleb-Minimum_24.pdf)

Valeriano, P. (1556). Hieroglyphica sive de sacris Aegyptiorum literis commentari. Basilea. Isingrin.

Varchi, B. (1549). Due lezzioni di messer Benedetto Varchi. Firenze: Lorenzo Torrentino [ma 1550].

Vellutello, A. (1560). Il Petrarca con l'espositione di Alessandro Vellutello. Di novo ristampato con le figure e $i$ Trionfi, con le apostille e con piu cose utili aggiunte. Venezia: Giolito.

Villari, S. (2013). Gli esordi della critica ariostesca. Lodovico Dolce e l'edizione del "Furioso" del 1535, Studi medievali e umanistici, 11, 119-174.

Zappella, G. (1986). Le marche dei tipografi e degli editori italiani del Cinquecento. Repertorio di figure, simboli e soggetti e dei relativi motti. Milano, Editrice bibliografica. 


\section{Apparato iconografico}

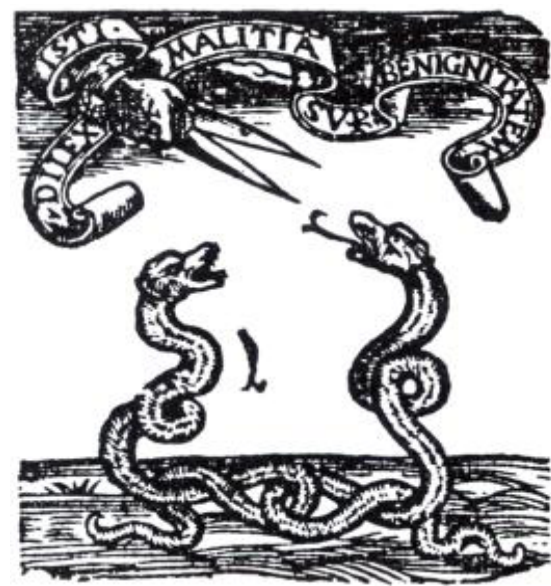

Fig. 1. Ariosto, L. (1532), marca tipografica (nel colophon, c. h8): Due serpenti intrecciati. In alto mano con forbice e motto. Cfr. ICCU, Edit16, CNCE 2566; Zappella, G. (1986), 1054; Fahy, C. (1989), tav. III.

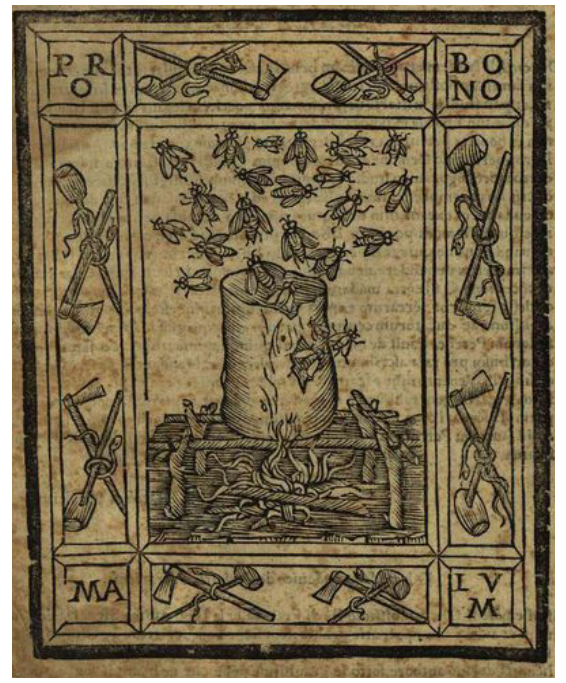

Fig. 2. Ariosto, L. (1516), c. a2v. Impresa con sciame d'api e motto. Cfr. ICCU, Edit16, CNCE 2541; Fahy, C. (1989), tav. I; Ariosto, L. (2006), p. 6.
"Mario Chi mandasse una biscia?

Cornelio Significherebbe malignità, alludendo a quel proverbio, che non si dee nudrire il serpe, né la [nella stampa] biscia in seno. Onde l'Ariosto essendo nella prima editione del suo Furioso morso dalla invidia de' detrattori, e di poi col tempo, havendo la verità come tagliata la lingua a que' maligni, conoscendosi il suo poema raro et eccellente, nella seconda $[s i c]$ editione levò questa impresa che fece stampare nella fine del libro: due biscie, all'una delle quali era stata tagliata la lingua, e all'altra, che gonfiata di veleno la vibrava, si mostrava di sopra una mano con una forbice in atto di tagliarla anco a lei, con un motto che dicea: DILEXISTI MALITIAM SUPER BENIGNITATEM".

Dolce, L. (1565a), c. 50r

"Che fu non meno bella impresa di quell'altra che pose nella prima sua editione subito nella prima carta; che fu un alveo di api, le quali dall'ingrato villano erano fatte fuggire col fuoco, quelle procacciando d'uccidere, quantunque elle [ella stampa] havessero prodotto il mele, ponendovi il motto PRO BONO MALUM". Dolce, L. (1565a), c. $50 \mathrm{rv}$ 


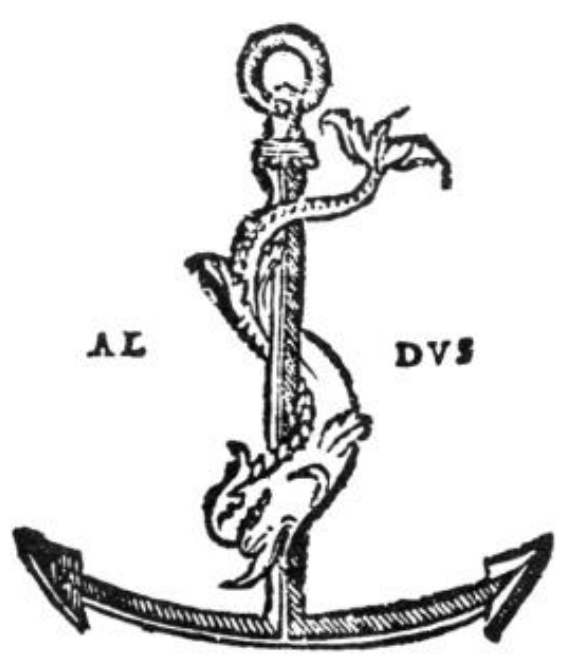

Fig. 3. Marca tipografica di Aldo Manuzio: Cft. ICCU. Edit16; Zappella, G. (1986), 1054, 38.
"Mario Chi mandasse un delfino?

Cornelio Il delfino è pesce velocissimo.

Onde dinoterebbe la prestezza.

MARIo Chi mandasse un'ancora?

Cornelio La fermezza, onde levò Tiberio quella bella impresa dell'ancora con delfino avoltovi a torno, con un motto, FESTINA LENTE, la quale impresa diede il Bembo, che solo una medaglia di lei n'haveva, a m. Aldo Romano, il quale la levò per insegna, et la usò poi sempre nei suoi libri”.

Dolce, L. (1565a), c. $54 r$

"[...] alcuni sono tanto veloci e precipitosi nelle attioni loro, che è troppo; ed altri così pegri $[$ sic] che rade volte fanno cosa che riesca bene. Bisognerebbe adunque che havessero in memoria il motto di Tiberio con la impresa del delfino e dell'ancora, e che lo ponessero in opra”.

Dolce, L. (1565a), c. $69 v$

\section{PATIENTIA ẼST ORNAMENTVM CVSTO DIA ET PROTECTIO VITAE.}

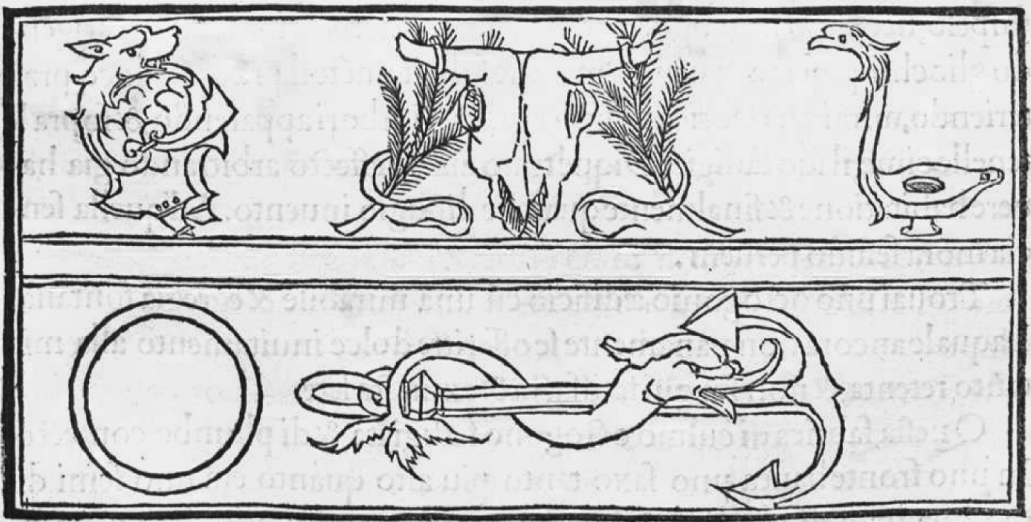

Dalaltra partetale elegäte fcal ptura mirai. Vnocirculo. Vna ancora Sopra la ftangula dillağle fe rouoluea uno Delphino. Et ạftioptimaméti cufi io li iterpretai. AFI $\Sigma \Pi$ I $\triangle$ E BPA $\triangle E \Omega \Sigma$. Semp feltina tarde.

Fig. 4 Xilografia dell' Hypnerotomachia Poliphili, Venezia, Aldo Manuzio Sr., 1499. Il motto greco è tradotto "Semper festina tarde" (cfr. ed. Pozzi-Chiappini, p. 61 e Bongiovanni, M.B., 2015, al sito https://www.bta.it/txt/a0/07/bta00771.html) 


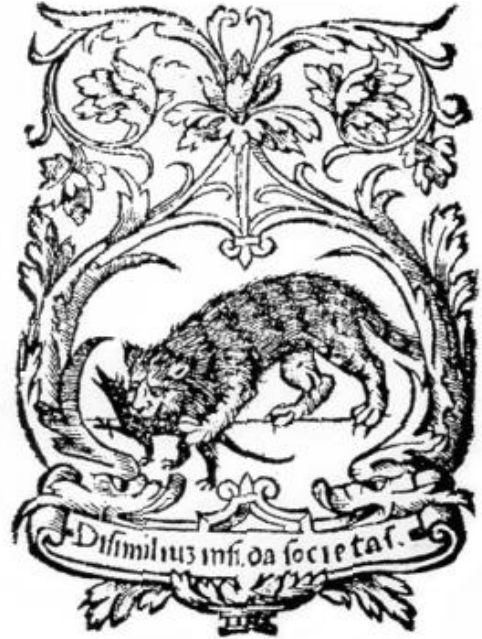

Fig. 5. Marca di Melchiorre Sessa: Gatta con topo in bocca, in cornice figurata, con motto. Cfr. ICCU, Edit16; Zappella, G. (1986), 592; Curi Nicolardi, S., p. 311, fig 12 .

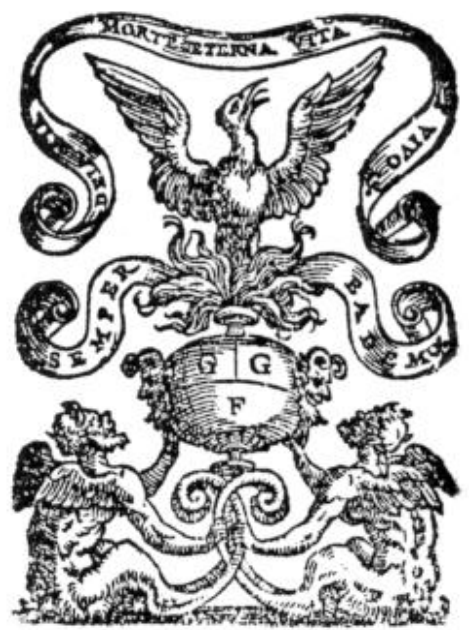

Fig. 6 Fenice su fiamme che si sprigionano da un'anfora, sorretta da due satiri alati, recante le iniziali G.G.F. Motti: "Semper eadem" e "De la mia morte eterna vita i vivo". Cfr. ICCU, Edit16; Zappella, G. (1986), 535. Cfr. fig. 7.
"Mario Chi mandasse a donare una gatta?

Cornelio La gatta mangia i topi, i quali sono di gran danno a una casa, percioché rodono cose di valore, come ornamenti di casa, libri e cose simili. E per questo si tengono nelle case, perché altrimenti apportano danno, rubando la carne, i pesci e rompendo sovente le massericie, oltre che hanno brutta effigie e sono ferocissime a guisa di leoni dei quali hanno certo sembiante. Onde potrebbe colui significare utile e parimente danno. E perché in qualunque casa honorata e civile, insieme con le gatte si tengono anco dei cani, tra i quali animali v'è battaglia sempre ordinaria, potrebbe anco significare che non vi può essere amicitia e concordia che duri se non tra pari. Onde l'honorato $\mathrm{m}$. Marchiò Sessa nella sua insegna, che è la gatta la quale tiene un topo in bocca, v'ha posto questo motto: DISSIMILIUM INFIDA SOCIETAS".

Dolce, L. (1565a), c. $56 v$

"[...] bella e convenevole insegna alla facultà delle lettere fu quella che levò il gentilissimo et honoratissimo signor Gabriello Giolito, essendo ella una fenice che arde nelle fiamme, risguardando incontra il sole, con questo motto: SEMPER EADEM; e volgarmente; De la mia morte eterna vita i' vivo sì che riferisce a quello: Vivo morte refecta mea, cioè "vivo rinata della morte mia". Onde non si poteva trovare più bella insegna, né più propria alle cose delle lettere, perché gl'impressori con l'imprimer de' libri tengono vivi i nomi degli scrittori e gli rendono immortali".

Dolce, L. (1565a), cc. 56v-57v 


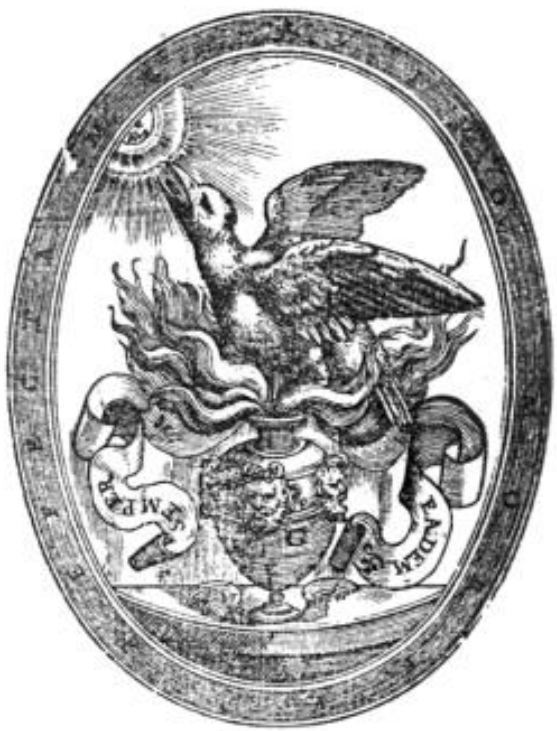

Fig. 7. Ovale posto al centro del frontespizio istoriato dell'edizione del Furioso del 1542 curata da Ludovico Dolce (Venezia: Giolito), con il motto sul contorno "Vivo morte refecta mea”. Cfr. ICCU, Edit16, CNCE 2628; Zappella, G. (1986), 540; Nuovo, A. - Coppens, C. (2005), fig. 27.

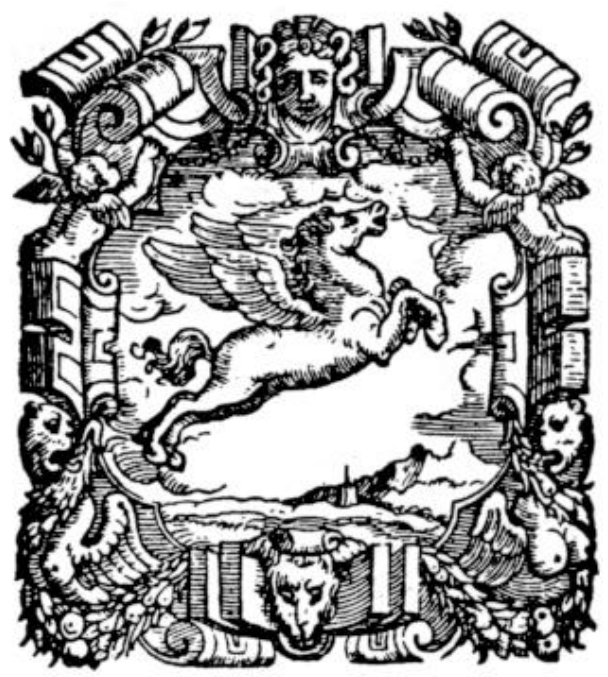

Fig. 8. Pegaso in volo; sullo sfondo città e montagne, in cornice figurata. Marca tipografica di Melchiorre Sessa. Cfr. ICCU, Edit16, CNCM 324; Zappella, G. (1986), 940 . 


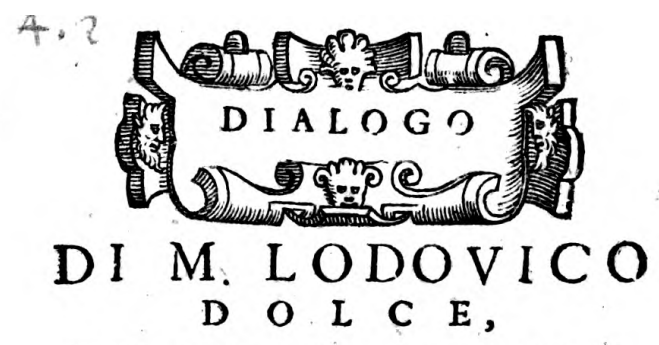

\section{NEL QVALE SI RAGIONA}

delle qualità, diuerfità, e proprietà de i colori.

CON P RIVILEGIO.

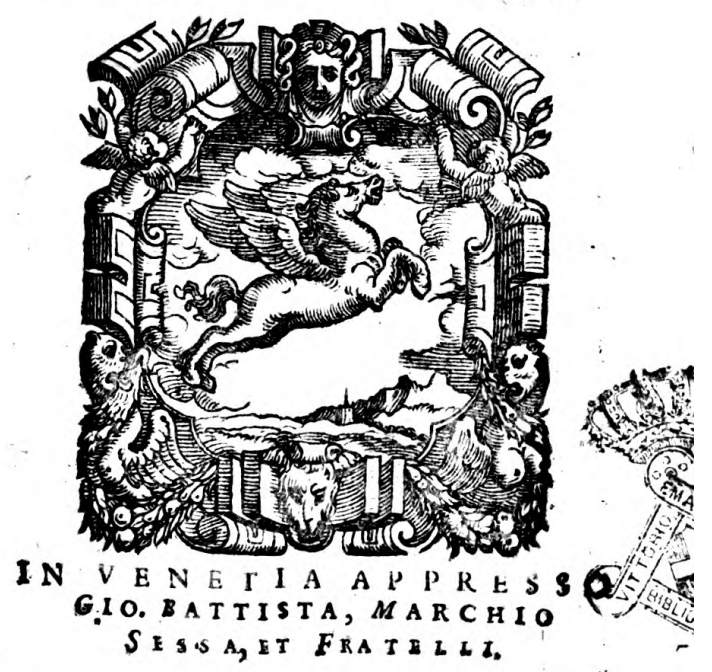

Fig. 9 Dolce, 1565a, frontespizio.

Esemplare conservato a Roma, Biblioteca nazionale centrale, 12.2.C.15.3 -digitalizzato in Google libri: https://books.google.it/books?id=0aCLf19q3qAC\&printsec=f rontcover\&hl=it\&source=gbs_ge_summary_r\&cad=0\#v=onepage\&q\&f=false. Cfr. ICCU, Edit16, CNCE 17387. 
mettic molto bizzarra , ef piena di uarii appettiti. Se mi fujpe opposfo, cbe net ipfalmo filegge, circum amilta uda rietate, come dire che lauarietà fe intende iui, come mo frano ghi fottili interpreti, de gli membri : cioe che al cas po, o dile brazze ; $\theta$ cofi di membro in memitro fidias no, li habiti conucnienti, (come diremmo) la beretta al capo, or cofi del refto : ne lodo molto quelli, che affettas no le fog gie de biabiti for affieri iparlo non tanto de colori, quanto del modo di ueftire, che tali fin da Plauto, $v$ Lu ciano fono detti Aagelli peregrini. Seruio di questo parla

Fig. 10. Morato, F.P. (1543), riproduzione parziale della c. D3r: "[...] Se mi fusse opposto che nel psalmo si legge circum amicta varietate, come dire che la varietà se intende ivi, come mostrano gli sottili interpreti, de gli membri, cioè che al capo et alle brazze et così di membro in membro si diano li habiti convenienti (come diremmo) la beretta al capo, et così del resto. Né [nella stampa: ne] lodo molto quelli che affettano le foggie de habiti forastieri, parlo non tanto de' colori, quanto del modo di vestire, che tali fin da Plauto et Luciano sono detti augelli peregrini [...]

piena diwari appetiti. E fe mi opponefti, che nel falmo fi legge circumamilfa uarietate; cioè lies ta e ueftita di uarietà:la uarietd s'intende iui, co me ipongonogl interprete : delle membra, ciod, che al capo $\mathbb{O}$ alle braccia, e cofi di membro in membro fi diano gli babiti conueneuoli: come disiamo noi la beretta al capoje cofi tel rimanenth

Fig. 10a. Dolce, L. (1565a). Riproduzione parziale della c. 36v: "[...] Et se mi opponesti che nel psalmo si legge circumamicta varietate, cioè lieta e vestita di varietà, la varietà si intende ivi, come ispongono gl'interprete, delle membre, cioè che al capo et alle braccia, et così di membro in membro, si diano gli habiti convevevoli, come diciamo noi la beretta al capo e così del rimanente." 


\section{DE I COLORI.}

Ma ftimo, che meriti lode colui, che affétta le foggie de gli babiti foraftieri e parlo non tanto de' colori, quanto del modo del ueftire, il quale bog

'gidi in I talia non è I taliano: perciocke, quando $f i$

: fanno ipanni alla Francele, quando alla spagnio

- la, quando alla $T$ edefé, e quarido al modo di una natione, equando d'altra, Onde bene diffe Dane

te, che uerrebbe il tempo

$\therefore$ Nel qual fard it pergaro interdetto

Alle sfacciate Donne Ficrentine

Andar moftrando con le poppe il petto .

Q uai barbare fur mai, quai faracine,

Cui bifognaffe, per far ir touerte oppiritali, o altre difcipline.

Cofibiafimaua Dante il corrotto ufo del ueftirfi,

-che era nel fuo tempo.Q uefti, che coji fecondo - Taltrui ufanza ueftano, fono chiamati da Plaut o da Luciano A ugelli peregrini, cioè foreftieri. Vo

Fig. 10b. Dolce, L. (1565a), Riproduzione parziale delle cc. $36 v$ e 37r: "Né [Ma nella stampa] stimo che meriti lode colui che affetta le foggie degli habiti forastieri, e parlo non tanto de' colori, quanto del modo del vestire, il quale hoggidì in Italia non è italiano; percioché, quando si fanno i panni alla francese, quando alla spagnuola, quando alla tedesca, e quando al modo di una natione, e quando d'altra. Onde bene disse Dante che verrebbe il tempo

Nel qual sarà in pergamo interdetto

Alle sfacciate donne fiorentine

Andar mostrando con le poppe il petto.

Quai barbare fur mai, quai saracine,

cui bisognasse, per far ir coverte

o spiritali, o altre discipline.

Così biasimava Dante il corrotto uso del vestirsi che era nel suo tempo. Questi che così, secondo l'altrui usanza, vestano sono chiamati da Plauto e da Luciano augelli peregrini, cioè forestieri [...]"

[nella citazione dantesca pergaro > pergamo è correzione dell'errata corrige: vd. fig. 14] 


\section{T A VO LA D E $\mathrm{E}^{87}$ \\ C $\mathrm{O} \quad \mathrm{L} \quad \mathrm{O} \quad \mathrm{R}$.}

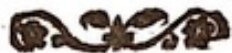

A

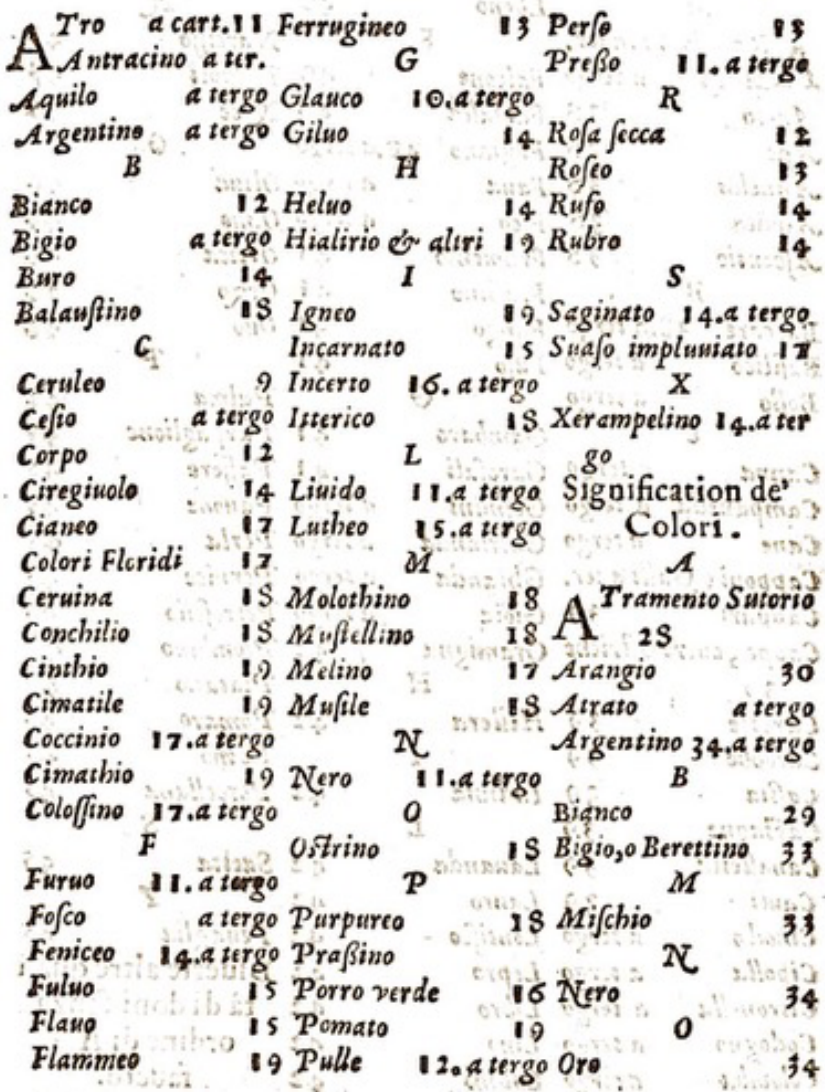

Fig. 11. Dolce, L. (1565a), c. 87r. Per l'esemplare: sopra, fig. 9 


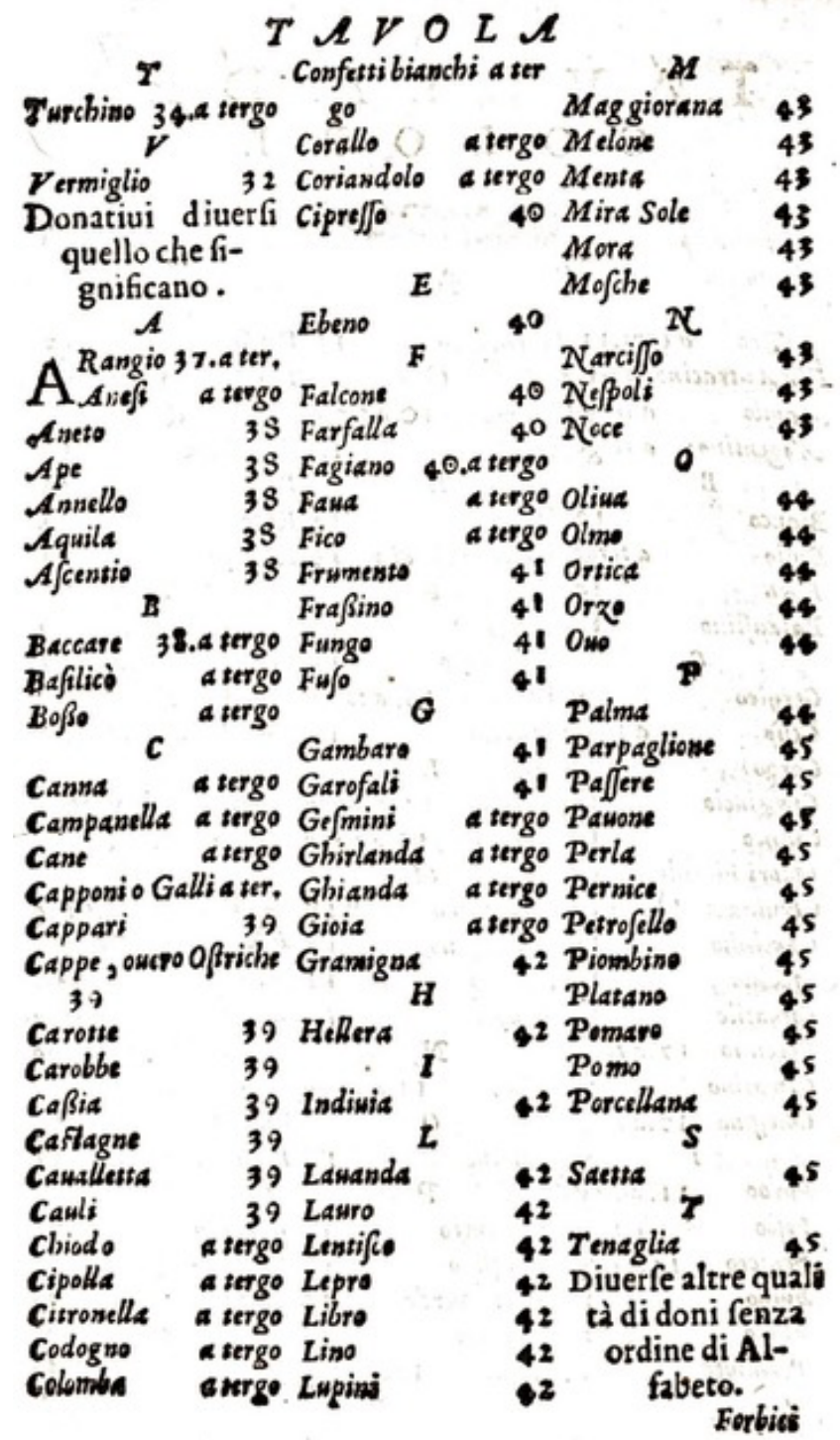

Fig. 12. Dolce, 1565 a, c. $87 v$ 


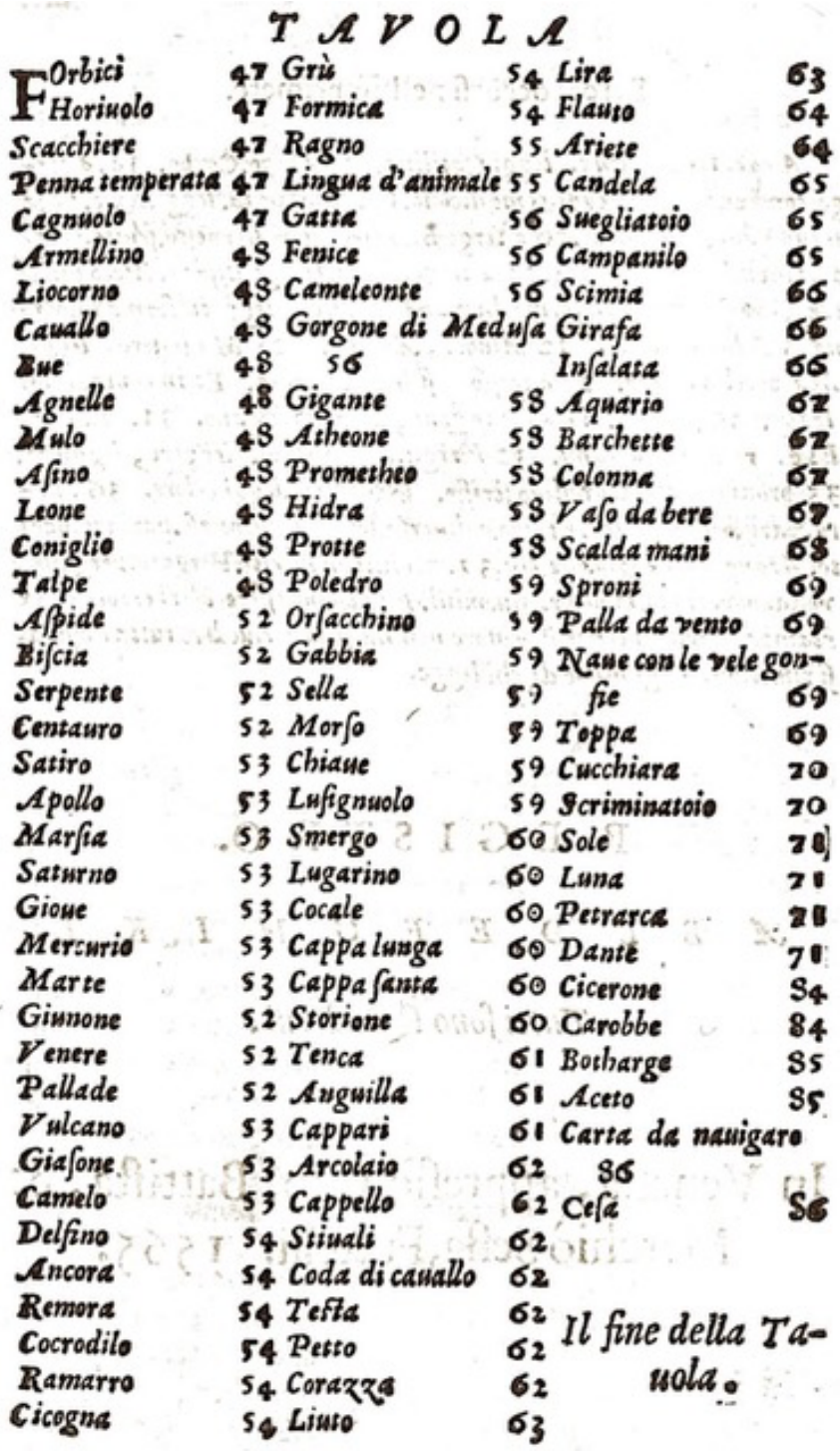

Fig. 13. Dolce, 1565a, c. [88]r. 
$\therefore$

Ertarị océcorfi nell'imprimefe.

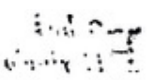

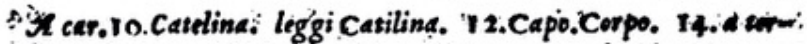

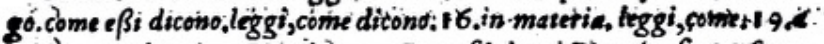

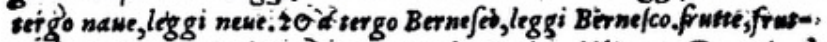

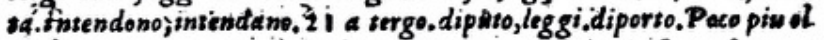

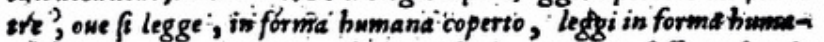

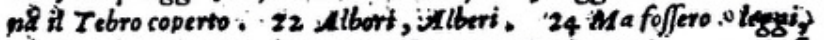
Ala perche fofjero. 29 a tergo. fi legge. Poni. Et in contrario $\hat{k}$ tegze. 30 a tergo, non fireggono, non fi veggano. 31 . Etcofes Ete. reccamt.raccami. 32 Virgili. Virgilio. .leggere, leggienti. 35. prodigo l'altra.prodigo fcrifje. $35.2 a l$ cofa.logigi colore. 36 . nam t5. Darÿ, wimno alorwi, ter go. o diverfa.legg gi fono diwer fo.parlare, pald

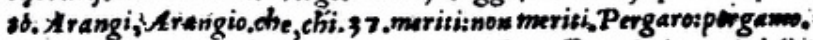

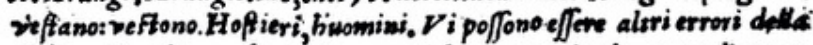

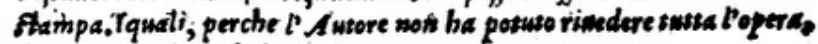
firithestonso al gindicio dis chi legge.

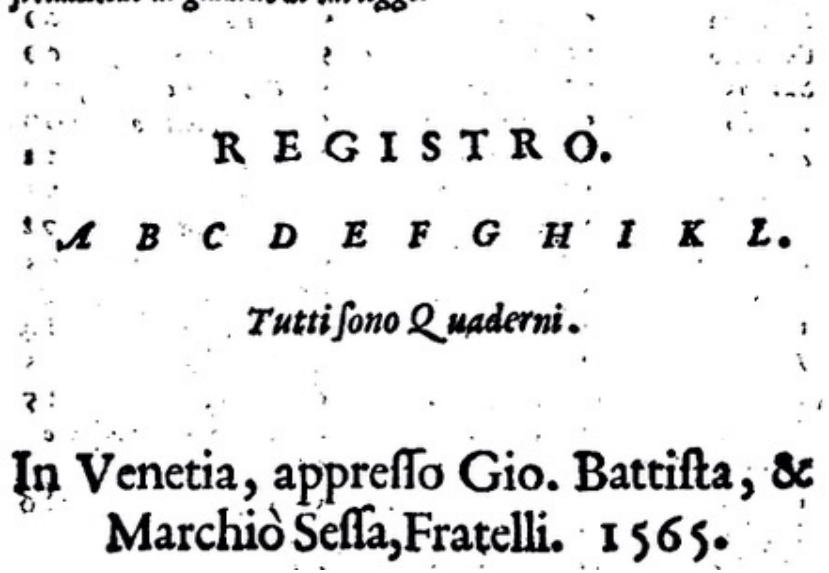

Fig. 14. Dolce, L. (1565a), errata corrige, registro e colophon. 


\section{-IL SIGNIFICATO DE MAZZOLLI}

de berbe of altre molte cofe, fitolle, o dal colore, 0 dallo odore, o dalla natura e uirts fue naturale,

: :; i o da qualche efteriore effetto o affetto o fis. militudine de noci come farebbe a dire.

Aglio amore Sporco, e purzzolente.

:Amandola, $\mathcal{\sigma}$ moraro,cioe amando il mio cupre moro.

Anarazzo,o perona, amore nó cóneniëte ad ambe le parti. Anefifcoperti;amore fen?a mal affetto.

Aneto, dolce amore, e fegreto, c casio e a buono fine, non lafriuo ne nergognofo.

Anime de frutti, defiderio di moftrare il core,e farficonos -. fcere in le vifiere o darfi in tutto e ptutto nó folamente uiuo ma doppò la diff ofitione del corpo a cui fi mäca. Annello, dar la fede a cui fi manda. Ape,uedi brefca.

Aquila, o penna, fignorile concupifenza.

Articbiocbi, aiutati al meglio che poi.

Afcenfio, fignifica a gl'affanni.

Bacara,nó temere, ne inuidıa, ne maladicêtia de malewoli.

Bafait oe, fojpetto e gelofia.

Bede, amore troppo fcortefe,e villane fco.

Betonega, niuna cofa e piu manifffla, herba de cui fretie fos no charofoli.

Borafo, berba, o fiori amore uniuer fale, non a per fona certa* Brefca di mele, ouero l'ape, che la fa detta percia fignifica piccbia pure, cioe batte; Sla faldo che uincerai.

Brogniuoli, non fi puo adefja.
D $\mathrm{iiii}$

Fig. 15a. Morato, F.P. (1543), c.D4r. 
colori è coja da pazzo. Ma jarebbemi grato, che apprefole altre cofe, dellequalliragionato $m$ ' bai, m infegnaßi la via d ifprimere diuerfi concetti, fe condo dı̣uerfità di colori, quando voglia me ne veniffe.

Co R. Farollo poienticri, fecödo, che a memoria mi uer rà.Ma perche di diuerfe forti d'berbe toglièdo ifi gnificati, cio $\{$ i $f a$, dall' odore, o dal colore, o dalla natura e virtù loro naturale; o da qualcbe efterio re effetto, ouero affetto, o fimiglianza di voci.

$M$ ar. Ne queftomi dipiace.

COR. Chi voleffe dimostrare, alcun'amore non effer conueneuole ad ambe le parti, potrebbe manda$r e$ ono arangio di fapore brufco; per hanere egli ona parte bella, che è la fcorza,e'l lapore non di letteuole.

MA R. Ilbrufco pecialméte fuol piacere ne gli arāgi.

$C \mathrm{O}$ : Basta, che infieme non conuengono: come fas rebbono, quando il fapor fofje dolce.

$M \mathrm{~A}$ R. Chi mailda Je in dono ad altrui ane $i$ fcopertis cioè non confetti, per quefto che cofa fignificareb.

Fig. 15b. Dolce, L. (1565a), riproduzione parziale della c. $37 v$. 
